\title{
An extension of the Landau-Ginzburg-de Gennes theory for liquid crystals $\dagger$
}

\author{
by L. LONGA $\ddagger$, D. MONSELESAN and H.-R. TREBIN \\ Institut für Theoretische und Angewandte Physik, Universität Stuttgart, \\ F.R. Germany
}

(Received 11 February 1987; accepted 8 June 1987)

\begin{abstract}
Using angular momentum representation a method is proposed that allows the systematic construction of a generalized Landau-de Gennes elastic free energy of liquid crystals, in powers of a symmetric and traceless tensor order parameter, polarization field, of external fields and all respective derivatives. By this method all linearly independent elastic invariants and surface terms are constructed for nematics and cholesterics up to fourth order terms. In particular it is shown that up to fourth order in the tensor order parameter there are nineteen bulk elastic constants and four surface terms in the free energy of a general, biaxial nematic. In addition, the stability of this expansion is studied in detail. Some special cases of the elastic free energy of liquid crystals, already discussed in the literature, are reexamined and discrepancies with our results are emphasized. Finally, a thermodynamically correct way of establishing contact between the generalized de Gennes elastic free energy and other theories, like those of Oseen-Frank or Meyer, is proposed by applying fluctuation theory. Thus, the degeneracy of splay and bend elastic constants is removed even when these are calculated from the standard de Gennes free energy. Restrictions on higher order elastic constants are also obtained by comparing mean field relations and stability conditions with available experimental data.
\end{abstract}

\section{Introduction}

Liquid crystals exhibit a rich variety of phases. Most extensively investigated are the isotropic, nematic, cholesteric, smectic A phases [1] and recently the blue phases [2]. Much interest has been focused on both elastic and thermal properties of the orientational degrees of freedom. It is widely accepted that the orientational properties of liquid crystals can be described by a second rank, symmetric, traceless tensor, $\mathbf{Q}(\mathbf{r})$ with cartesian components $Q_{\alpha \beta}(\mathbf{r})(\alpha, \beta=x, y, z)$. The tensor vanishes in the isotropic phase and thus serves as an order parameter. In the ordered phases nematic, cholesteric, etc., $\mathbf{Q}(\mathbf{r})$ has uniaxial symmetry. The symmetry axis is defined by an eigenvector, $\hat{n}(\mathbf{r}),\left(\hat{n}^{2}=1\right)$ of $\mathbf{Q}(\mathbf{r})$, corresponding to the only non-degenerate eigenvalue. In the most general case $\mathbf{Q}(\mathbf{r})$ has five independent components. This situation describes the so-called general biaxial phase [3]. A spatial dependence of $\mathbf{Q}$ requires elastic terms in the Landau free energy expansion. Together with the thermal contribution the elastic terms form the orientational part of the free energy of liquid crystals.

Historically, the first steps toward our present understanding of the elastic properties of liquid crystals were made by Oseen [4], Zocher [5] and Frank [6] (OZF). The

† Supported in part by Deutsch Forschungsgemeinschaft.

$\ddagger$ Alexander von Humboldt Foundation Fellow, 1985-1987. Permanent address: Jagellonian University, Department of Statistical Physics, Reymonta 4, Kraków, Poland. 
OZF description consists of a sum of five functions in the director and its derivatives multiplied by elastic constants (material parameters) $K_{i j}$

$$
\begin{aligned}
F= & \int d^{3} \mathbf{r}\left[\frac{1}{2} K_{11}(\nabla \cdot \hat{n})^{2}+\frac{1}{2} K_{22}\left(\hat{n} \cdot \nabla \times \hat{n}-q_{0}\right)^{2}+\frac{1}{2} K_{33}(\hat{n} \times \nabla \times \hat{n})^{2}\right. \\
& \left.+\frac{K_{22}+K_{24}}{2}\left(n_{\alpha, \beta} n_{\beta, \alpha}-n_{\alpha, \alpha} n_{\beta, \beta}\right)\right] .
\end{aligned}
$$

In this and succeeding expressions a subscript after a comma denotes differentiation with respect to the corresponding cartesian coordinate. The free energy (1) contains only terms that are symmetric with respect to a change of $\hat{n}$ into $-\hat{n}$, a restriction now widely accepted by many authors for weakly polar liquid crystals. As already discussed by Frank [6], the elastic constants $K_{i i}$ measure the resistance of three simple types of distortion, called splay, twist and bend, respectively while $q_{0}$ is known as the helicity.

In general, the parameters $K_{i j}$ and $q_{0}$ depend on temperature, pressure and other thermodynamic variables. For some phases, like smectics, the number of relevant elastic constants can be reduced significantly. It is also worth mentioning that, in the expansion (1), the term proportional to $\left(K_{22}+K_{24}\right)$ is often omitted because it can be converted to a surface integral by applying Gauss's theorem. However, for some types of boundary value problems [7] and in the presence of defects [8] the surface term does contribute to the total free energy.

The list of formal properties of the OZF expansion must be completed with inequalities among the elastic constants that follow from the stability analysis of (1). As discussed by Ericksen [9], the $K$ s are subject to the relations

$$
K_{11} \geqslant 0, K_{22} \geqslant\left|K_{24}\right|, K_{33} \geqslant 0,2 K_{11}-K_{22}-K_{24} \geqslant 0,
$$

which ensure the internal consistency of the theory. They must be fulfilled in any experiment if the theory is complete. Though much effort has been expended to obtain the numerical values of the elastic constants [10-12], it is still difficult to discuss the consistency of the theory as dictated by the inequalities (2) since there are no independent measurements of $K_{24}$. Some authors resort to the assumption of Nehring and Saupe [13] that

$$
2 K_{24}=K_{11}-K_{22},
$$

which, by inequality (2), requires that

$$
3 K_{11} \geqslant K_{22} \text {. }
$$

The existing experimental data [10-12] are generally consistent with the inequality $(3 b)$, except near the nematic-smectic phase transition.

In order to generalize the OZF description close to the clearing point, de Gennes [14] proposed a Landau-Ginzburg type of expansion for $F$ in terms of the tensor order parameter $\mathbf{Q}$ and its derivatives $Q_{\alpha \beta, \gamma}$. In the absence of electric and magnetic fields, the original expression for $F$ reads

$$
\begin{aligned}
F= & F_{0}+a\left(T-T^{*}\right) \operatorname{Tr} \mathbf{Q}^{2}+B \operatorname{Tr} \mathbf{Q}^{3} \\
& +C\left(\operatorname{Tr} \mathbf{Q}^{2}\right)^{2}+\cdots \\
& +L_{1}^{(2)} Q_{\alpha \beta, \gamma} Q_{\alpha \beta . \gamma}+L_{2}^{(2)} Q_{\alpha \beta, \beta} Q_{\alpha \gamma, \gamma} \\
& +L_{4}^{(2)} \varepsilon_{\alpha \beta \gamma} Q_{\alpha \varrho} Q_{\beta Q, \gamma} .
\end{aligned}
$$


The parameters $a, B, C, L_{i}^{(2)}(i=1,2,4)$ are now assumed to be independent of temperature $T$, and $\varepsilon_{\alpha \beta \gamma}$ denotes the Levi-Civita tensor. In addition summation over repeated indices is to be understood if not stated otherwise. The last term in equation $(4 a)$ violates parity and is responsible for the formation of a helical ground state. The equivalent term in expression (1) is proportional to $q_{0} K_{22}$. A further second order term in $Q_{\alpha \beta, \gamma}$

$$
L_{3}^{(2)} Q_{\alpha \beta, \gamma} Q_{\alpha \gamma, \beta}
$$

was omitted by de Gennes because it can be written as a linear combination of a surface term and the elastic terms already included in expansion $(4 a)$.

De Gennes's theory, as expressed by equation $(4 a)$, gives very simple expressions for the OZF elastic constants. However, as pointed out by Lubensky [15] and de Jeu [12], the expansion ( $4 a$ ) implies $K_{11}=K_{33}$, in clear contradiction to experiment. This degeneracy of the elastic constants cannot be removed even by adding the missing term $(4 b)$ to the expansion ( $4 a$ ). A possible way out of this difficulty is to include higher order terms, like $\mathbf{Q}(\partial \mathbf{Q})(\partial \mathbf{Q})$ where $\mathbf{Q}(\partial \mathbf{Q})(\partial \mathbf{Q})$ denotes the class of all independent $\mathrm{SO}(3)$-invariants built up from the tensor $Q_{\alpha \beta} Q_{\delta \varepsilon, \gamma} Q_{\mu v, \varrho}$. Such a programme has been carried out partially, by Schiele and Trimper [16] for nematics and has further been generalized by Berremann and Meiboom [17] and Poniewierski and Sluckin [18]. In [17] the OZF elastic constants are calculated under the assumption that $\mathbf{Q}$ is uniaxial

$$
Q_{\alpha \beta}(\mathbf{r})=S\left(n_{\alpha}(\mathbf{r}) n_{\beta}(\mathbf{r})-\frac{\delta_{\alpha \beta}}{3}\right)
$$

In third order of the expansion the degeneracy of $K_{11}$ and $K_{33}$ is removed. A linear dependence of $K_{i i} / S^{2}$ on $S$ has been predicted, but Berremann and Meiboom had to introduce, ad hoc, an additional term to the energy proportional to $S^{4} /(1-S)^{2}$ to achieve agreement with experiment for the temperature dependence of all OZF elastic constants. The assumed form, however, of the extra term is not justified by the analysis presented.

A different solution to the problem of the degeneracy of elastic constants was proposed by Rościszewski [19] who showed that instead of including third order terms into Landau-Ginzburg-de Gennes theory, one could equivalently introduce lowest order elastic terms in additional order parameters, for example, a fourth rank tensor $R_{\alpha \beta \gamma \delta}$. This theory is especially attractive because it introduces only two new elastic constants and shows the importance of an order parameter such as $\left\langle P_{4}\right\rangle$, i.e. the average value of the fourth order Legendre polynomial.

In this paper we show that there exists yet another way to remove the degeneracy of $K_{11}$ and $K_{33}$. All of the theories mentioned are seriously limited by the assumption that the order parameter is strictly uniaxial and that its modulus $S$ is constant. As pointed out by Poniewierski and Sluckin [18] this assumption may not be true in the neighbourhood of disclinations, close to a phase transition or at the interface between a solid substrate and a liquid crystal. More importantly, the general third order theory in $Q_{\alpha \beta}$ is unstable with respect to biaxial fluctuations and thus is thermodynamically incorrect. Fourth order terms have to be included (or neglect third order ones) to preserve the stability of the free energy.

Furthermore the generalizations of the OZF theory in $[17,18]$ overestimate the number of independent invariants in the general biaxial case. The authors use 
cartesian tensors, but the cartesian representation gives only an upper limit for the number of independent terms because the constraints

$$
Q_{\alpha \alpha}=0, \quad Q_{\alpha \alpha, \beta}=0 \quad(\forall \beta)
$$

cannot be easily incorporated into the cartesian picture. For instance, there are only six invariants of the form $\mathbf{Q}(\partial \mathbf{Q})(\partial \mathbf{Q})$ while $[17,18]$ suggest seven. Also, cartesian representation suggests sixteen invariants of the form $\mathbf{Q} \mathbf{Q}(\partial \mathbf{Q})(\partial \mathbf{Q})$ while only thirteen are linearly independent. Overestimations of the number of independent invariants can be found elsewhere [26].

Up to now we have paid no attention to the influence of molecular polarity on the thermodynamic properties of liquid crystals. The necessity of including these effects was suggested long ago by Meyer [20]. But only recently has a considerable amount of evidence become available that strong permanent dipoles may lead to qualitatively new phenomena, like the occurrence of antiferroelectric, smectic A phases or re-entrant phase transitions [21]. An influence of molecular dipole moments on the properties of blue phases has also been indicated by Stegemeyer et al. [2]. The evidence motivates the introduction of another order parameter in the description of the orientational properties of liquid crystals, namely the polarization density $\mathbf{P}(\mathbf{r})$.

Theoretical studies of polar effects in terms of $\mathbf{P}(\mathbf{r})$ were initialized by Meyer [20] with his theory of flexoelectricity in nematics. He predicted that liquid crystals exhibit a special type of flexoelectricity linked with curvature of the orientation pattern. Recently, Meyer's theory has been extended by Barbero et al. [22] to include effects at the solid-liquid crystal interfaces. In these models the polarity is an induced effect, strictly related to the molecular shape. A number of papers has also been devoted to studies of spontaneous ferroelectricity in chiral systems [23, 24] and nematics [25]. Among these is the paper of Khachaturyan [25], which discusses the structure of a hypothetical ferroelectrical nematic phase. Khachaturyan shows that if a nematic ferroelectric liquid crystal with the symmetry group $C_{\infty \circ v}$ exists, then such a ferroelectric will be inhomogeneous with wide domain walls, which are similar to the helical structure.

Finally, the Landau-de Gennes theory has been investigated with inclusion of external fields, both electric and magnetic. Although the basic theory is well established [14], it has been generalized only recently to include some higher order cross couplings between electric and magnetic fields and the order parameter $\mathbf{Q}$ [26]. These couplings are relevant because many of the applications of liquid crystals depend on their ability to respond strongly to external stimuli. It is also interesting to note that in some cases, like the blue phases, the response is difficult to predict [2].

Our concern will be to construct a thermodynamically correct, lowest order expansion of the orientational part of the liquid crystal free energy in terms of $\mathbf{Q}$, the gradient of $\mathbf{Q}$ (symbolized by $\partial \mathbf{Q}$ ), $\mathbf{P}, \partial \mathbf{P}, \mathbf{E}$ and $\mathbf{H}$ which

(a) removes the degeneracy of the OZF elastic constants;

(b) contains only independent invariants; and

(c) is, at least for nematics with zero polarization, stable with respect to general biaxial fluctuations.

Stability conditions of the expansion will be analysed in detail for nematic symmetry. Their implications on the elastic constants will be discussed. In particular, it will be shown by explicit calculations that there are twenty-two bulk elastic constants and 
four surface terms in the elastic energy of general nematics described in terms of $\mathbf{Q}$. In addition a systematic study of the surface terms and surface relations will be given. In order to find all linearly independent invariants, all surface terms and stability conditions, systematic use will be made of the spherical representation for tensor and vector fields. This method provides us with an invariant expansion that leads to the simplest stability criteria. The final formulas will be converted to a cartesian picture as this allows a compact notation. A connection between standard director and tensor pictures is also discussed, that appears as a consequence of applying thermodynamic rules. Finally, estimation of various elastic constants from experimental data is given.

The organization of this paper is as follows. In $\S 2$ some basic rules for the calculation of invariants and surface relations are presented. In $\S 3$ the general theory for systems with nematic and cholesteric symmetries is developed. In $\S 4$ the stability of the expansion is discussed for non-polar nematics. Section 5 is devoted to systematic studies of relations between the director and the tensor pictures. In particular formulas for the OZF elastic constants are derived and the importance of thermodynamic fluctuations is emphasized. In $\$ 6$ the connection between the generalized Landau-de Gennes and the Meyer theories is explored. Section 7 gives an estimation of some of tensor elastic couplings. Section 8 closes the paper with a short summary.

\section{General theory}

To establish invariant polynomials in the components of the traceless symmetric tensor field $\mathbf{Q}(\mathbf{r})$, of the polarization vector field $\mathbf{P}(\mathbf{r})$, and of the gradients of both fields, we transform from cartesian to spherical representation. The spherical components of $\mathbf{Q}$ form an $L=2$ quadrupole tensor $Q_{m}^{(2)}(m= \pm 2, \pm 1,0)$, where

$$
\left.\begin{array}{l}
Q_{ \pm 2}^{(2)}=-\frac{1}{2}\left(Q_{x x}-Q_{y y} \pm 2 i Q_{x y}\right), \\
Q_{ \pm 1}^{(2)}= \pm\left(Q_{x z} \pm i Q_{y z}\right), \\
Q_{0}^{(2)}=\frac{3}{\sqrt{6}}\left(Q_{x x}+Q_{y y}\right) .
\end{array}\right\}
$$

The spherical components of any vector field or vector operator $\mathbf{A}$ form an $L=1$ dipole tensor $A_{m}^{(1)}(m= \pm 1,0)$, where

$$
\left.\begin{array}{l}
A_{ \pm 1}^{(1)}=\mp \frac{i}{\sqrt{2}}\left(A_{x} \pm i A_{y}\right), \\
A_{0}^{(1)}=i A_{z} .
\end{array}\right\}
$$

In the spherical representation all components $Q_{m}^{(2)}$ are independent, and the constraints $Q_{\alpha \beta}=Q_{\beta \alpha}, \operatorname{Tr} \mathbf{Q}=0$ of the cartesian representation do not have to be taken into account.

From the components $T_{m}^{(l)}$ and $S_{q}^{(k)}$ of two spherical tensors of ranks $l$ and $q$, respectively, $(2 l+1)(2 k+1)$ products $T_{m}^{(l)} S_{q}^{(k)}$, which are reducible, can be formed. 'By forming linear combinations weighted by Clebsch-Gordan coefficients, we can form $(2 l+1)(2 k+1)$ irreducible components transforming according to the angular quantum number $L \in\{l+k, l+k-1, \ldots|l-k|\}$

$$
\left[T^{(l)} \otimes S^{(k)}\right]_{M}^{(L)}:=\sum_{m_{1}, m_{2}}\left(\begin{array}{cc|c}
l & k & L \\
m_{1} & m_{2} & m
\end{array}\right) T_{m_{1}}^{(l)} S_{m_{2}}^{(k)},
$$


Table 1. Symmetry properties of basic fields used in the construction of the LandauGinzburg expansion.

\begin{tabular}{lcc}
\hline & \multicolumn{2}{c}{ Sign after } \\
Tensor & Time reversal & Space inversion \\
\hline $\mathbf{Q}$ & + & + \\
$\mathbf{V}$ & + & - \\
$\mathbf{P}$ & + & - \\
$\mathbf{E}$ & + & - \\
$\mathbf{H}$ & - & + \\
\hline
\end{tabular}

where $\left(\begin{array}{cc|c}l & k & L \\ m_{1} & m_{2} & m\end{array}\right)$ are the Clebsch-Gordan coefficients. If $T$ and $S$ are identical, then of course permutation symmetry has to be observed, and this reduces the number of independent (irreducible and reducible) components. To the product $\left[T^{(l)} \otimes S^{(k)}\right]^{(L)}$ we can couple a third tensor $R_{s}^{(p)}$ to form a spherical product $\left[\left[T^{(l)} \otimes S^{(k)}\right]^{(L)} \otimes R^{(p)}\right]^{(K)}$.

The independent invariant polynomials in the components of the vector fields or vector operators $\mathbf{A}=\boldsymbol{\nabla}, \mathbf{P}, \mathbf{E}, \mathbf{H}$, and the quadrupole tensor $\mathbf{Q}$, are now constructed by coupling the spherical components to all possible $L=0$ (i.e. $\mathrm{SO}(3)$-invariant) tensors, which are symmetric under time reversal (i.e. containing only even powers of $\mathbf{H}$, see table 1) and which are not conversed mutually by the permutation operation. As example for the independent invariants quadratic in $\partial_{\gamma} \mathbf{Q}_{\alpha \beta}$, we couple $\partial^{(1)}$ and $Q^{(2)}$ to

$$
\left[\partial^{(1)} \otimes Q^{(2)}\right]^{(I)} \equiv \partial Q^{(I)}, \quad I=1,2,3,
$$

and then couple these mutually to $K=0$. The only possibilities are

$$
\begin{aligned}
I_{2}(L)= & {\left[\left[\partial^{(1} \otimes Q^{(2)}\right]^{(L)} \otimes\left[\partial^{(1)} \otimes Q^{(2)}\right]^{(L)}\right]^{(0)}, } \\
= & \sum_{m=-L}^{L}\left(\begin{array}{cc|c}
L & L & 0 \\
m & -m & 0
\end{array}\right)\left(\partial^{(1)} \otimes Q^{(2)}\right)_{m}^{(L)} \\
& \times\left(\partial^{(1)} \otimes Q^{(2)}\right)_{-m}^{(L)}, \quad L=1,2,3 .
\end{aligned}
$$

The spherical representation offers two important advantages:

(i) The linear independence of Clebsch-Gordan coupled invariants is immediately evident.

(ii) In many cases (as in our example) the invariants appear as positive definite quantities and allow an easy discussion of the stability of the free energy expansion.

As an example we write down the lowest order elastic terms of the free energy density in the basis $I_{2}(L)$, from equation $(8 a)$

$$
F=\sum_{L=1}^{3} K_{L}^{(2)} I_{2}(L)
$$

It is seen that because $I_{2}(L) \geqslant 0$, the free energy $(8 b)$ is stable if the elastic constants $K_{L}^{(2)}$ are positive. Thus the spherical representation gives the simplest stability conditions, $K_{L}^{(2)} \geqslant 0$. 
Another problem, that can be studied systematically by use of spherical representation is the identification of so-called surface relations. By definition, surface relations are linear combinations of invariants which can be expressed as a full divergence. Thus, if due to suitable boundary conditions surface terms vanish, certain invarian $\mathrm{s}$ become linearly dependent. This allows for further simplification of the free energy expansion. The number of surface relations is found in a systematic way as follows. First, the number of independent divergence terms associated with a given set of invariants is found using spherical representation. For instance, the invariants in equation $(8 a)$ are combined to surface terms via equation

$$
\partial_{\gamma}\left(Q_{\alpha \beta} Q_{\mu v, \varrho}\right)=Q_{\alpha \beta, \gamma} Q_{\mu \nu, \varrho}+Q_{\alpha \beta} Q_{\mu \nu, \ell \gamma} .
$$

It is straightforward to write a similar relation in the spherical representation using the correspondence

$$
\partial_{\gamma}\left(Q_{\alpha \beta} Q_{\mu v, \varrho}\right) \leftrightarrow \partial^{(1)} \otimes\left(Q^{(2)} \otimes \partial^{(1)} \otimes Q^{(2)}\right)
$$

and

$$
Q_{\alpha \beta} Q_{\mu \nu, e \gamma} \leftrightarrow Q^{(2)} \otimes \partial^{(1)} \otimes \partial^{(1)} \otimes Q^{(2)} .
$$

According to equation (9) each divergence term can be written as a linear combination of the invariants and some higher order terms, like $Q_{\alpha \beta} Q_{\mu v, Q y}$ or equivalently $Q^{(2)} \otimes \partial^{(1)} \otimes \partial^{(1)} \otimes Q^{(2)}$. Now suppose that the number of independent divergence terms is $k$, the number of invariants is $l$ and the number of higher order invariants generated by divergence terms is $n(k \leqslant n+l)$. By taking linear combinations of various divergence terms we obtain new $(k-n)$ full divergence terms which are expressed as linear combinations of invariants and in which higher order invariants do not appear. They are precisely the surface relations we are looking for. Thus, the number of surface relations is given by the difference $k-n$. Returning to our previous example we find that there are three independent invariants in the tensor space $\partial^{(1)} \otimes\left(Q^{(2)} \otimes \partial^{(1)} \otimes Q^{(2)}\right)$ and two higher order invariants $Q^{(2)} \otimes \partial^{(1)} \otimes \partial^{(1)} \otimes Q^{(2)}$. Consequently, there exists only one surface relation built up of the invariants $(8)$ that allows us to eliminate the term $(4 b)$ from the free energy expansion $(4 a)$, as originally done by de Gennes. Although the spherical representation easily solves the problem of the number of linearly independent invariants and surface relations, the most easily understood form for the free energy is obtained in the cartesian representation. Hence, to construct the free energy expansion, we proceed in the following way:

(i) first, we calculate the number of linearly independent invariants and surface relations using the spherical representation;

(ii) next, we look for the corresponding set of cartesian invariants and extract from this set linearly independent terms; if this is difficult the linear independence of invariants is studied using computer programs for algebraic manipulation;

(iii) finally, we study the stability of the free energy in the spherical representation and transfer the results to the cartesian representation.

Furthermore, all terms appearing in the free energy expansion are classified with respect to space reversal transformations as $\mathrm{SO}(3)$ or $\mathrm{O}(3)$ invariants. The space inversion divides all invariants into scalars (nematic symmetry) and pseudoscalars 
(cholesteric symmetry). A full list of sign changes of basic tensors used to construct the free energy expansion is given in table 1.

\section{Invariant expansion of the free energy}

In the preceding section we showed a systematic way, with which to construct the Landau-Ginzburg-de Gennes expansion for the free energy to arbitrary order. As we have seen from a simple example, a higher order approximation requires careful elimination of the constrains $Q_{\alpha \alpha}=Q_{\alpha \alpha, \gamma}=0$, and so we propose to use the spherical representation for the tensor fields. Statements about the number of independent invariants are listed in table 2 . We now discuss some of these results in a more detailed manner. First, we define the orientational free energy as a sum of bulk and elastic terms

$$
\int_{V} d^{3} r\left[F_{\mathrm{bulk}}+F_{\mathrm{N}, \mathrm{el}}+F_{\mathrm{ch}, \mathrm{el}}+F_{\mathrm{p}, \mathrm{el}}+F_{\mathrm{ext}, \mathrm{el}}\right]
$$

where $F_{\text {bulk }}$ is given by

$$
\begin{aligned}
F_{\text {bulk }}= & a\left(T-T^{*}\right) \operatorname{Tr} \mathbf{Q}^{2}+B \operatorname{Tr} \mathbf{Q}^{3}+C\left[\operatorname{Tr} \mathbf{Q}^{2}\right]^{2} \\
& +D\left[\operatorname{Tr} \mathbf{Q}^{2}\right]\left[\operatorname{Tr} \mathbf{Q}^{3}\right]+E\left[\operatorname{Tr} \mathbf{Q}^{2}\right]^{3} \\
& +E^{\prime}\left[\operatorname{Tr} \mathbf{Q}^{3}\right]^{2}-\frac{1}{2} \Delta \chi_{\max } M_{\alpha} Q_{\alpha \beta} M_{\beta}-\frac{1}{2} \Delta \varepsilon_{\max } E_{\alpha} Q_{\alpha} E_{\beta} \\
& +\mu_{1} P^{2}+\mu_{2} P^{4}+\nu P_{\alpha} Q_{\alpha \beta} P_{\beta}+\varepsilon_{1} \mathbf{P E} \\
& +\varepsilon_{2} P_{\alpha} Q_{\alpha \beta} E_{\beta}+\cdots
\end{aligned}
$$

and where the terms $F_{\mathrm{N}, \mathrm{el}}, F_{\mathrm{ch}, \mathrm{el}}, F_{\mathrm{p}, \mathrm{el}}, F_{\text {ext,el }}$ represent elastic contributions with nematic, chiral, polar, and external fields couplings, respectively. Apart from the standard terms the bulk free energy also contains lowest order couplings with the polarization field $\mathbf{P}$, and with external fields. These extra terms (together with gradient terms discussed later in this paper) could in principle modify phase diagrams for nematics, cholesterics and blue phases, a possibility which will be discussed in a forthcoming publication. Our concern now is to study meticulously the form of elastic terms in the expansion (10).

\subsection{Elastic free energy of nematic liquid crystals}

The expansion of the free energy in powers of $Q_{\alpha \beta}$ and its derivatives up to the order $\mathbf{Q} \mathbf{Q} \partial \mathbf{Q} \partial \mathbf{Q}$, contains twenty-two terms and four surface relations (see table 2). They are

$\partial Q \partial Q[14]:$

$$
\left.\begin{array}{l}
L_{1}^{(2)} Q_{\alpha \beta, \gamma} Q_{\alpha \beta, \gamma}, \\
L_{2}^{(2)} Q_{\alpha \beta, \beta} Q_{\alpha \gamma, \gamma}, \\
L_{3}^{(2)} Q_{\alpha \beta, \gamma} Q_{\alpha \gamma, \beta}
\end{array}\right\}
$$

$Q \partial Q \partial Q[16-18]:$

$$
\left.\begin{array}{ll}
L_{1}^{(3)} Q_{\alpha \beta} Q_{\alpha \beta, \mu} Q_{\mu v, v}, & L_{2}^{(3)} Q_{\alpha \beta} Q_{\alpha \mu, \beta} Q_{\mu v, v}, \\
L_{3}^{(3)} Q_{\alpha \beta} Q_{\alpha \mu, \mu} Q_{\beta v, v}, & L_{4}^{(3)} Q_{\alpha \beta} Q_{\alpha \mu, v} Q_{\beta \mu, v}, \\
L_{5}^{(3)} Q_{\alpha \beta} Q_{\alpha \mu, \nu} Q_{\beta v, \mu}, & L_{6}^{(3)} Q_{\alpha \beta} Q_{\alpha \mu, v} Q_{\mu v, \beta} .
\end{array}\right\}
$$


Table 2. Number of invariants and surface relations in the generalized Landau-Ginzburg-de Gennes theory. We denote with an asterisk the numbers that are different from those cited in the literature.

\begin{tabular}{lccccc}
\hline $\begin{array}{l}\text { Invariants composed } \\
\text { of } \mathbf{Q} \text { and } \partial \mathbf{Q}\end{array}$ & $\begin{array}{c}\text { Surface } \\
\text { terms }\end{array}$ & $\begin{array}{c}\text { Inversion } \\
\text { symmetry }\end{array}$ & $\begin{array}{c}\text { Number of } \\
\text { invariants }\end{array}$ & $\begin{array}{c}\text { Number of } \\
\text { surface } \\
\text { terms }\end{array}$ & $\begin{array}{c}\text { Number of } \\
\text { surface } \\
\text { relations }\end{array}$ \\
\hline$\partial \mathbf{Q} \partial \mathbf{Q}[14]$ & $\partial[\mathbf{Q} \partial \mathbf{Q}]$ & + & 3 & 3 & 1 \\
$\mathbf{Q} \partial \mathbf{Q} \partial \mathbf{Q}[16-18]$ & $\partial[\mathbf{Q Q} \partial \mathbf{Q}]$ & + & $6^{*}$ & 5 & 1 \\
$\mathbf{Q} \mathbf{Q} \partial \mathbf{Q} \partial \mathbf{Q}$ & $\partial[\mathbf{Q Q Q} \partial \mathbf{Q}]$ & + & 13 & 7 & 2 \\
$\mathbf{Q} \partial \mathbf{Q}[14,17]$ & - & - & 1 & - & - \\
$\mathbf{Q Q} \partial \mathbf{Q}[14,17]$ & - & - & 1 & - & - \\
$\mathbf{Q Q Q} \partial \mathbf{Q}$ & - & - & 2 & - & - \\
\hline
\end{tabular}

Invariants composed of

$\mathbf{P}, \mathbf{Q}, \mathbf{E}$ and $\mathbf{H} \quad$ Inversion symmetry Number of invariants

\begin{tabular}{|c|c|c|c|c|c|c|}
\hline $\mathbf{P}$ & $\mathbf{P}$ & & & & + & 1 \\
\hline $\mathbf{P}$ & $\mathbf{P}$ & $\mathbf{Q}$ & & & + & 1 \\
\hline $\mathbf{P}$ & $\mathbf{P}$ & $\mathbf{Q}$ & $\mathbf{Q}$ & & + & 2 \\
\hline E & $E$ & $\mathbf{Q}$ & $\mathbf{Q}$ & [26] & + & 2 \\
\hline H & H & $\mathbf{Q}$ & $\mathbf{Q}$ & {$[26]$} & + & 2 \\
\hline $\mathbf{P}$ & E & & & & + & 1 \\
\hline $\mathbf{P}$ & $\mathbf{Q}$ & E & & & + & 1 \\
\hline $\mathbf{P}$ & $\mathbf{Q}$ & $\mathbf{Q}$ & E & & + & 2 \\
\hline $\mathbf{P}$ & $\mathbf{Q}$ & $\mathbf{Q}$ & Q & $E$ & + & 2 \\
\hline
\end{tabular}

Invariants composed of $\mathbf{Q}, \partial \mathbf{Q}, \mathbf{P}, \partial \mathbf{P}$, $\mathbf{E}$ and $\mathbf{H}$ Surface terms

Inversion Number of

Number of Number of symmetry invariants surface surface

terms relations

\begin{tabular}{|c|c|c|c|c|c|c|c|}
\hline E & $\partial \mathbf{Q}$ & [26] & $\partial(\mathbf{E}$ & Q) & + & 1 & 1 \\
\hline E & $\mathbf{Q}$ & $\partial \mathbf{Q} \quad[26]$ & $\partial(\mathbf{E}$ & QQ) & + & $3^{*}$ & 2 \\
\hline E & $\mathbf{Q}$ & $\mathbf{Q} \partial \mathbf{Q}$ & $\partial(\mathbf{E}$ & QQQ) & + & 5 & 2 \\
\hline $\begin{array}{l}P \\
Q\end{array}$ & $\begin{array}{l}\partial \mathbf{Q} \\
\partial \mathbf{P}\end{array}$ & & $\partial[\mathbf{Q}$ & P] & $\begin{array}{l}+ \\
+\end{array}$ & $\begin{array}{l}1 \\
1\end{array}$ & 1 \\
\hline$P$ & $\mathbf{Q}$ & $\partial \mathbf{Q}$ & $\partial[\mathbf{P}$ & $00]$ & + & 3 & 2 \\
\hline $\mathbf{Q}$ & $\mathbf{Q}$ & $\partial \mathbf{P}$ & & & + & 2 & \\
\hline $\begin{array}{l}\mathbf{P} \\
\mathbf{Q}\end{array}$ & $\begin{array}{l}\mathbf{Q} \\
\mathbf{O}\end{array}$ & $\begin{array}{ll}\mathbf{Q} & \partial \mathbf{Q} \\
\mathbf{O} & \partial \mathbf{P}\end{array}$ & $\partial[\mathbf{P C}$ & 200] & $\begin{array}{l}+ \\
+\end{array}$ & $\begin{array}{l}5 \\
2\end{array}$ & 2 \\
\hline$\partial \mathbf{P}$ & $\partial \mathbf{P}$ & & $\partial[\mathbf{P}$ & $\partial \mathbf{P}]$ & + & 3 & 3 \\
\hline $\mathbf{Q}$ & $\partial \mathbf{Q}$ & $\partial \mathbf{P}$ & $\partial[\mathbf{Q}$ & $\mathbf{Q} \quad \partial \mathbf{P}]$ & - & 7 & 8 \\
\hline$P$ & $\partial \mathbf{Q}$ & $\partial \mathbf{Q}$ & $\partial[\mathbf{Q}$ & $\left.\begin{array}{ll}\mathbf{P} & \partial \mathbf{Q}\end{array}\right]$ & - & 2 & \\
\hline $\begin{array}{l}\mathrm{E} \\
\mathrm{E}\end{array}$ & $\begin{array}{l}\mathbf{Q} \\
\mathbf{P}\end{array}$ & $\begin{array}{l}\partial \mathbf{P} \\
\partial \mathbf{Q}\end{array}$ & $\partial[\mathbf{E}$ & $\begin{array}{ll}\mathbf{Q} & \mathbf{P}]\end{array}$ & - & $\begin{array}{l}2 \\
2\end{array}$ & 2 \\
\hline E & E & $\partial \mathbf{Q} \quad[26]$ & $\partial[\mathbf{E}$ & E $\quad$ Q] & - & 1 & 1 \\
\hline H & H & $\partial \mathbf{Q}[26]$ & $\partial[\mathbf{H}$ & $\left.\begin{array}{ll}\mathbf{H} & \mathbf{Q}\end{array}\right]$ & - & 1 & 1 \\
\hline $\mathbf{P}$ & $\partial \mathbf{P}$ & & & - & - & 1 & - \\
\hline $\begin{array}{l}\mathbf{P} \\
\mathbf{P}\end{array}$ & $\begin{array}{l}\mathbf{Q} \\
\mathbf{P}\end{array}$ & $\begin{array}{l}\partial \mathbf{P} \\
\partial \mathbf{P}\end{array}$ & $\partial[\mathbf{P}$ & $\left.\begin{array}{ll}P & 0\end{array}\right]$ & - & $\begin{array}{l}2 \\
1\end{array}$ & 1 \\
\hline$\partial \mathbf{Q}$ & $\partial \mathbf{P}$ & & $\begin{array}{l}\partial[\mathbf{Q} \\
\partial[\mathbf{P}\end{array}$ & $\begin{array}{c}\partial \mathbf{P}] \\
\partial \mathbf{Q}]\end{array}$ & - & 2 & $\begin{array}{l}2 \\
2\end{array}$ \\
\hline
\end{tabular}


$Q Q \partial Q \partial Q$

Surface relations:

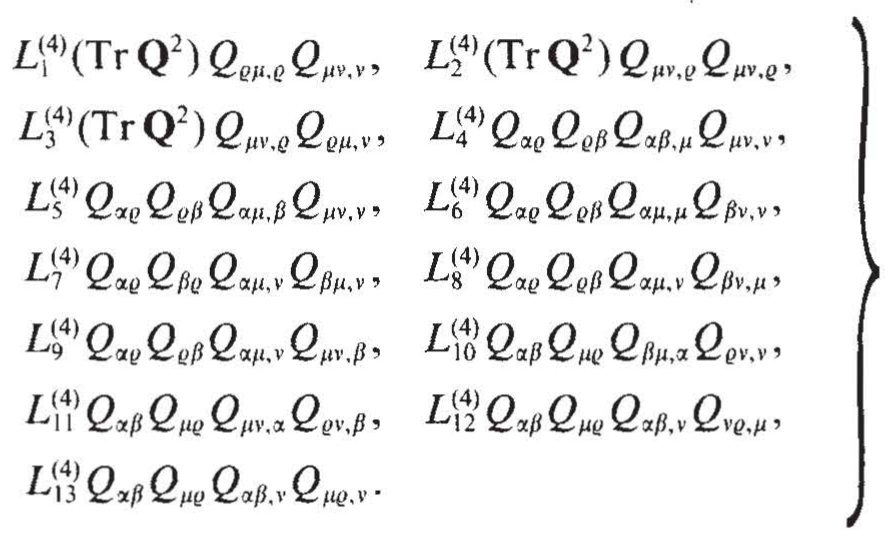

$$
\begin{gathered}
\partial_{\alpha}\left[Q_{\alpha \beta} Q_{\beta \gamma, \gamma}-Q_{\beta,} Q_{\alpha \beta, v}\right]=\left[L_{2}^{(2)}\right]-\left[L_{3}^{(2)}\right][14], \\
\partial_{v}\left[Q_{\alpha \beta} Q_{\alpha \mu} Q_{\beta v, \mu}\right]-\partial_{\beta}\left[Q_{\alpha \beta} Q_{\alpha \mu} Q_{\mu v, v}\right] \\
=\left[L_{5}^{(3)}\right]+\left[L_{6}^{(3)}\right]-\left[L_{2}^{(3)}\right]-\left[L_{3}^{(3)}\right][18], \\
\partial_{Q}\left[Q_{Q \mu} Q_{\alpha \beta} Q_{\alpha \beta} Q_{\mu v, v}-Q_{\mu \nu} Q_{\alpha \beta} Q_{\alpha \beta} Q_{e \mu, v}\right] \\
=-\left[L_{3}^{(4)}\right]-2\left[L_{4}^{(4)}\right]+2\left[L_{5}^{(4)}\right]+2\left[L_{6}^{(4)}\right]+2\left[L_{10}^{(4)}\right]-2\left[L_{12}^{(4)}\right] \\
\partial_{v}\left[Q_{\beta e} Q_{\alpha \alpha} Q_{\mu \nu} Q_{\alpha \mu, \beta}\right]-\partial_{\beta}\left[Q_{\beta e} Q_{Q \alpha} Q_{\mu \nu} Q_{\alpha \mu, v}\right] \\
=-\left[L_{1}^{(4)}\right] / 2+\left[L_{3}^{(4)}\right] / 2+3\left[L_{5}^{(4)}\right]-3\left[L_{9}^{(4)}\right] .
\end{gathered}
$$

Here $\left[L_{n}^{(m)}\right]$ denotes the invariant weighted by the elastic constant $L_{n}^{(m)}$. The proof of the independence of the invariants $\left[L_{n}^{(3)}\right]$ and $\left[L_{n}^{(4)}\right]$ is given in the Appendix. Keeping track of the mutual dependence is important, because the number of cartesian invariants one can form by different full contractions exceeds the number of really independent invariants. For instance, the often cited term [17, 18]

$$
L_{7}^{(3)} Q_{\alpha \beta} Q_{\mu v, \alpha} Q_{\mu v, \beta}
$$

can, in fact, be expressed as a linear combination of $\left[L_{n}^{(3)}\right](n=1,6)$,

$$
\left[L_{1}^{(3)}\right]-\left[L_{2}^{(3)}\right]-\left[L_{3}^{(3)}\right] / 2+\left[L_{4}^{(3)}\right]-\left[L_{5}^{(3)}\right] / 2-\left[L_{6}^{(3)}\right]+\left[L_{7}^{(3)}\right] / 2=0 .
$$

Note that the relation $(13 f)$ between seven invariants $\left[L_{n}^{(3)}\right](n=1,7)$ is not obvious and is rather tedious to obtain.

\subsection{Elastic free energy with purely chiral terms}

Up to fourth order in $Q$ there are four chiral terms linear in the gradient of $Q$ which are readily determined by the method described in $\S 2$,

$$
\left.\begin{array}{l}
L_{4}^{(2)} \varepsilon_{\alpha \beta \gamma} Q_{\alpha \varrho} Q_{\gamma e, \beta} \quad[14,17], \\
L_{7}^{(3)} \varepsilon_{\alpha \beta \gamma} Q_{\alpha \mu} Q_{\beta \nu} Q_{\mu y, \nu}, \\
L_{14}^{(4)} \varepsilon_{\alpha \beta \gamma} Q_{\alpha \mu} Q_{\beta \nu} Q_{v e} Q_{\mu e, \gamma}, \\
L_{15}^{(4)} \varepsilon_{\alpha \beta \gamma}\left(\operatorname{Tr} \mathbf{Q}^{2}\right) Q_{\alpha \varrho} Q_{\gamma e, \beta} .
\end{array}\right\}
$$

In deriving the number of fourth order terms use has been made of the decomposition of the representation $Q^{(2)} \otimes Q^{(2)} \otimes Q^{(2)}$ into a direct sum of irreducible representations

$$
Q^{(2)} \otimes Q^{(2)} \otimes Q^{(2)}=D^{0} \oplus D^{2} \oplus D^{3} \oplus D^{4} \oplus D^{6},
$$


where $D^{L}=\left[Q^{(2)} \otimes Q^{(2)} \otimes Q^{(2)}\right]^{(L)}$ is the irreducible representation corresponding to angular momentum $L$.

\subsection{Elastic free energy with polarization field included}

This group of terms is new. Again it can be divided into scalar- and pseudoscalar couplings as indicated in table 2 . They are listed together with respective surface relations

$P \partial Q, Q \partial P$ :

Surface relation:

$$
\left.\begin{array}{l}
A_{1}^{(2)} P_{\alpha} Q_{\alpha \beta, \beta}, \quad A_{2}^{(2)} Q_{\alpha \beta} P_{\beta, \alpha} . \\
\partial_{\alpha}\left[P_{\beta} Q_{\alpha \beta}\right]=\left[A_{1}^{(2)}\right]+\left[A_{2}^{(2)}\right] .
\end{array}\right\}
$$

$P Q \partial Q, Q Q \partial P$

Surface relations:

$$
\begin{array}{ll}
A_{1}^{(3)} Q_{\alpha \beta} P_{\alpha} Q_{\beta \gamma, \gamma}, & A_{2}^{(3)} Q_{\alpha \beta} P_{\gamma} Q_{\alpha \beta, \gamma}, \\
A_{3}^{(3)} Q_{\alpha \beta} P_{\gamma} Q_{\gamma \beta, \alpha}, & A_{4}^{(3)} \operatorname{Tr}\left(\mathbf{Q}^{2}\right) P_{\alpha, \alpha}, \\
A_{5}^{(3)} Q_{\alpha \beta} Q_{\beta \gamma \gamma} P_{\gamma, \alpha} . &
\end{array}
$$

$$
\begin{aligned}
\partial_{\alpha}\left[\operatorname{Tr}\left(Q^{2}\right) P_{\alpha}\right] & =2\left[A_{1}^{(3)}\right]+\left[A_{4}^{(3)}\right] \\
\partial_{\alpha}\left[Q_{\alpha \beta} Q_{\beta \gamma} P_{\gamma}\right] & =\left[A_{3}^{(3)}\right]-\left[A_{1}^{(3)}\right]+\left[A_{5}^{(3)}\right] .
\end{aligned}
$$

$P Q Q \partial Q, Q Q Q \partial P$

Surface relations:

$$
\begin{array}{ll}
A_{1}^{(4)} P_{\alpha} Q_{\alpha \beta} Q_{\gamma \delta} Q_{\gamma \delta, \beta}, & A_{2}^{4} P_{\alpha} Q_{\alpha \beta} Q_{\gamma \delta} Q_{\beta \delta, \gamma}, \\
A_{3}^{(4)} P_{\alpha} Q_{\beta \gamma} Q_{\gamma \delta} Q_{\beta \delta, \alpha}, & A_{4}^{(4)} P_{\alpha} Q_{\alpha \beta} Q_{\beta \gamma} Q_{\gamma \delta, \delta}, \\
A_{5}^{(4)} P_{\alpha} \operatorname{Tr}\left(\mathbf{Q}^{2}\right) Q_{\alpha \beta, \beta}, & A_{6}^{(4)} \operatorname{Tr}\left(\mathbf{Q}^{2}\right) Q_{\alpha \beta} P_{\beta, \alpha}, \\
A_{7}^{(4)} \operatorname{Tr}\left(\mathbf{Q}^{3}\right) P_{\alpha, \alpha} . &
\end{array}
$$

$$
\begin{aligned}
& \partial_{\alpha}\left[Q_{\beta \gamma} Q_{\beta \gamma} Q_{\alpha \delta} P_{\delta}\right]=2\left[A_{1}^{(4)}\right]+\left[A_{5}^{(4)}\right]+\left[A_{6}^{(4)}\right], \\
& \partial_{\alpha}\left[Q_{\beta \gamma} Q_{\gamma \delta} Q_{\delta \beta} P_{\alpha}\right]=3\left[A_{3}^{(4)}\right]+\left[A_{7}^{(4)}\right] .
\end{aligned}
$$

$\partial P \partial P$ :

Surface relation:

$$
\left.\begin{array}{l}
B_{1}^{(2)} P_{\alpha, \beta} P_{\alpha, \beta}, \quad B_{2}^{(2)} P_{\alpha, \alpha} P_{\beta, \beta}, \quad B_{3}^{(2)} P_{\alpha, \beta} P_{\beta, \alpha} \cdot \\
\partial_{\alpha}\left[P_{\alpha} P_{\beta, \beta}-P_{\beta} P_{\alpha, \beta}\right]=\left[B_{2}^{(2)}\right]-\left[B_{3}^{(2)}\right] .
\end{array}\right\}
$$

This list can be completed with the chiral terms

$P \partial P$ :

$$
C_{\mathrm{l}}^{(2)} \varepsilon_{\alpha \beta \gamma} P_{\alpha} \partial_{\beta} P_{\gamma} .
$$


$\partial Q \partial P$

Surface relation:

$$
\left.\begin{array}{c}
C_{2}^{(2)} \varepsilon_{\alpha \beta \gamma} P_{\beta, \alpha} Q_{\gamma \delta, \delta}, C_{3}^{(2)} \varepsilon_{\alpha \beta \gamma} P_{\alpha, \delta} Q_{\gamma \delta, \beta}, \\
\varepsilon_{\alpha \beta \gamma}\left\{\partial_{\alpha}\left[P_{\beta} Q_{\gamma \delta, \delta}\right]+\partial_{\delta}\left[P_{\alpha} Q_{\gamma \delta, \beta}\right]\right\}=\left[C_{2}^{(2)}\right]+\left[C_{3}^{(2)}\right] .
\end{array}\right\}
$$

$P Q \partial P, P P \partial Q$

Surface relation:

$$
\begin{aligned}
& C_{1}^{(3)} \varepsilon_{\alpha \beta \gamma} P_{\delta} Q_{\gamma \delta} P_{\beta, \alpha}, \quad C_{2}^{(3)} \varepsilon_{\alpha \beta \gamma} P_{\beta} Q_{\gamma \delta} P_{\delta, \alpha} \\
& C_{3}^{(3)} \varepsilon_{\alpha \beta \gamma} P_{\beta} P_{\delta} Q_{\gamma \delta, \alpha} .
\end{aligned}
$$

$$
\varepsilon_{\alpha \beta \gamma} \partial_{\alpha}\left[P_{\beta} P_{\delta} Q_{\gamma \delta}\right]=\left[C_{1}^{(3)}\right]+\left[C_{2}^{(3)}\right]+\left[C_{3}^{(3)}\right] .
$$

$Q \partial Q \partial P, P \partial Q \partial Q$

$$
\begin{array}{ll}
C_{4}^{(3)} \varepsilon_{\alpha \beta \gamma} P_{\delta} Q_{\alpha \varrho, \varrho} Q_{\gamma \delta, \beta}, & C_{5}^{(3)} \varepsilon_{\alpha \beta \gamma} P_{\delta} Q_{\beta \varrho, \alpha} Q_{\varrho \delta, \gamma}, \\
C_{6}^{(3)} \varepsilon_{\alpha \beta \gamma} Q_{\delta \varrho} P_{\gamma, \beta} Q_{\alpha \delta, \varrho}, & C_{7}^{(3)} \varepsilon_{\alpha \beta \gamma} Q_{\alpha \delta} P_{\gamma, \beta} Q_{\delta \varrho, \varrho}, \\
C_{8}^{(3)} \varepsilon_{\alpha \beta \gamma} Q_{\delta \varrho} P_{\varrho, \beta} Q_{\alpha \delta, \gamma}, & C_{9}^{(3)} \varepsilon_{\alpha \beta \gamma} Q_{\alpha \delta} P_{\varrho, \beta} Q_{\delta \varrho, \gamma}, \\
C_{10}^{(3)} \varepsilon_{\alpha \beta \gamma} Q_{\delta \varrho} P_{\gamma, \beta} Q_{\delta \varrho, \alpha}, & C_{11}^{(3)} \varepsilon_{\alpha \beta \gamma} Q_{\alpha \delta} P_{\varrho, \delta} Q_{\gamma \varrho, \beta}, \\
C_{12}^{(3)} \varepsilon_{\alpha \beta \gamma} Q_{\alpha \delta} P_{\varrho, \beta} Q_{\gamma \delta, \varrho} . &
\end{array}
$$

Surface relations:

$$
\begin{aligned}
\varepsilon_{\alpha \beta \gamma} \partial_{\gamma}\left[Q_{\alpha \varrho} Q_{\varrho \delta} P_{\delta, \beta}\right] & =\left[C_{8}^{(3)}\right]+\left[C_{9}^{(3)}\right], \\
\varepsilon_{\alpha \beta \gamma,} \partial_{\alpha}\left[\operatorname{Tr} Q^{2} P_{\beta, \gamma}\right] & =2\left[C_{10}^{(3)}\right], \\
\varepsilon_{\alpha \beta \gamma} \partial_{\alpha}\left[Q_{\beta \varrho} P_{\sigma} Q_{\varrho \sigma, \gamma}\right] & =\left[C_{5}^{(3)}\right]-\left[C_{9}^{(3)}\right] .
\end{aligned}
$$

3.4. Elastic couplings with external fields

For this group the dominant contributions are of the form

$$
E \partial Q \quad[26], E Q \partial Q, E Q Q \partial Q:
$$

This group of terms can be obtained by replacing $P_{\alpha}$ with $E_{\alpha}$ in equation $(15 a)-(15 c)$. Additionally we have cross-coupling terms:

$E Q \partial P, E P \partial Q$

Surface relations:

$$
\left.\begin{array}{cc}
\zeta_{1}^{(3)} \varepsilon_{\alpha \beta \gamma} E_{\varrho} Q_{\varrho \alpha} P_{\gamma, \beta}, & \zeta_{2}^{(3)} \varepsilon_{\alpha \beta \gamma} E_{\alpha} Q_{\beta \varrho} P_{\gamma, \varrho}, \\
\zeta_{3}^{(3)} \varepsilon_{\alpha \beta \gamma} E_{\varrho} P_{\gamma} Q_{\varrho \alpha, \beta}, & \zeta_{4}^{(3)} \varepsilon_{\alpha \beta \gamma} E_{\alpha} P_{\gamma} Q_{\beta \varrho, \varrho} . \\
& \\
\varepsilon_{\alpha \beta \gamma} \partial_{\beta}\left[E_{\varrho} Q_{\varrho \alpha} P_{\gamma}\right]=\left[\zeta_{1}^{(3)}\right]+\left[\zeta_{3}^{(3)}\right], \\
\varepsilon_{\alpha \beta \gamma} \partial_{\varrho}\left[E_{\alpha} Q_{\beta \varrho} P_{\gamma}\right]=\left[\zeta_{2}^{(3)}\right]+\left[\zeta_{4}^{(3)}\right] .
\end{array}\right\}
$$

EE $\partial Q \quad[26]:$

$$
\zeta_{s}^{(3)} \varepsilon_{\alpha \beta \gamma} E_{\alpha} E_{\varrho} \partial_{\beta} Q_{\gamma \varrho}
$$


There exists a term analogous to $(17 c)$ for the coupling of $\mathbf{Q}$ with an external magnetic field [26].

4. Stability of the nematic expansion for $P=E=H=0$.

General relations between the elastic constants can be obtained in the form of inequalities from the requirement that the elastic free energy expansion be stable with respect to order parameter fluctuations. As an example, let us consider first the case of a nematic phase whose elastic properties can be described by means of $F_{\mathrm{N} \text {, el }}$, where $F_{\mathrm{N}, \text { el }}$ is the linear combination of invariants $\left[L_{i}^{(2)}\right], i=1,2,3$. Because invariants $\left[L_{i}^{(2)}\right]$ are bilinear forms in $Q_{\alpha \beta, \gamma}$, the free energy $F_{\mathrm{N} \text {, el }}$ is a bilinear from in fifteen-dimensional space, spanned by independent fields $Q_{\alpha \beta, \gamma}$. Thus, the stability requirement of $F_{\mathrm{N}, \mathrm{el}}$ can be translated into the positive definiteness of the bilinear form. On the other hand, the positive definiteness of the bilinear form is most easily studied in the basis that diagonalizes the matrix of the form. In this basis the stability requirement means that all eigenvalues of the matrix of the form are positive numbers.

The advantage of using the spherical representation in finding stability criteria is that the matrix of the form is diagonal in independent variables $\partial Q_{m}^{(L)}, L=1,2,3$; $m=-L, \ldots, L$

$$
\begin{aligned}
F_{\mathrm{N}, \mathrm{el}} & =\sum_{\alpha=1}^{3} L_{\alpha}^{(2)}\left[L_{\alpha}^{(2)}\right]=\sum_{J=1}^{3} K_{J}^{(2)} I_{2}(J) \\
& =\sum_{J=1}^{3} K_{J}^{(2)} \frac{1}{\sqrt{ } 2 J+1} \sum_{m=-J}^{J}\left|\partial Q_{m}^{(J)}\right|^{2}
\end{aligned}
$$

Thus, $F_{\mathrm{N}, \text { el }}$, equation ( $18 a$ ), written as the sum of independent, positive definite terms, leads to a simple set of inequalities, $K_{L}^{(2)} \geqslant 0, L=1,2,3$ which preserves its stability. These inequalities can also be rewritten in an equivalent form by using the elastic constants $L_{\alpha}^{(2)}$. Replacing $\left[L_{\alpha}^{(2)}\right]$ in equation $(18 a)$ by linear combinations of $I_{2}(\beta)$ (see the Appendix) and extracting the coefficients $K_{\beta}^{(2)}$ gives

$$
\left.\begin{array}{l}
L_{1}^{(2)}+\frac{5}{3} L_{2}^{(2)}+\frac{1}{6} L_{3}^{(2)} \geqslant 0, \\
L_{1}^{(2)}-\frac{1}{2} L_{3}^{(2)} \geqslant 0, \\
L_{1}^{(2)}+L_{3}^{(2)} \geqslant 0 .
\end{array}\right\}
$$

Conditions $(18 b)$ are necessary and sufficient for the free energy expansion (18a) to be stable.

Qualitatively different inequalities are obtained by assuming that for all physically relevant fields the surface energies are negligible in the thermodynamic limit. For instance, the relation between second order terms

$$
\begin{aligned}
& \lim _{V \rightarrow \infty} \frac{1}{V} \int_{V} d^{3} x \partial_{\beta}\left[Q_{\alpha \beta} Q_{\alpha \gamma, \gamma}-Q_{\alpha \beta, \gamma} Q_{\gamma, \alpha}\right] \\
& =\lim _{V \rightarrow \infty} \frac{1}{V} \int_{V} d^{3} x\left(\left[L_{2}^{(2)}\right]-\left[L_{3}^{(2)}\right]\right)=0,
\end{aligned}
$$

leads to, $\left[L_{2}^{(2)}\right] \equiv\left[L_{3}^{(2)}\right]$, where the equivalence relation $\equiv$ means equality up to the surface terms. This, in turn, allows us to eliminate $\left[L_{3}^{(2)}\right]\left(\right.$ or $\left.I_{2}(3)\right)$ from the free energy 
expansion (18a). In this case, the relations (18b) give simpler inequalities [14],

$$
L_{1}^{(2)} \geqslant 0, \quad L_{1}^{(2)}+\frac{2}{3} L_{2}^{(2)} \geqslant 0, \quad \text { with } L_{3}^{(2)}=0 .
$$

This analysis does not apply directly to higher orders of expansion of $F_{\mathrm{N} \text {, el }}$. To address the problem more specifically, let us investigate the stability conditions for the fourth order expansion of $F_{\mathrm{N} \text {, el }}$, given by

$$
F_{\mathrm{N}, \mathrm{el}}=\sum_{i=1}^{3} L_{i}^{(2)}\left[L_{i}^{(2)}\right]+\sum_{i=1}^{6} L_{i}^{(3)}\left[L_{i}^{(3)}\right]+\sum_{i=1}^{13} L_{i}^{(4)}\left[L_{i}^{(4)}\right] .
$$

The expansion (19) is a polynomial of fourth order in independent fields $Q_{\alpha \beta}$ and $Q_{\alpha \beta, \gamma}$. Thus the problem of stability of expansion (19) is now reduced to a study of the properties of fourth order polynomials in the twenty-dimensional space of $Q_{\alpha \beta}$ and $Q_{\alpha \beta, \gamma}$. This appears to be a rather difficult problem. Still using the notion of bilinear forms, a simple analysis could be given that allows us to determine sufficient conditions for the free energy (19) to be stable. Namely, let us rewrite expansion (19) as a sum of expressions that are full squares. To do so, we note that expansion, in equation (19), could be considered as a general, bilinear form in the variables $Q_{\alpha \beta, \gamma}, Q_{\alpha \beta} Q_{\gamma \delta, \varrho}$. The easiest way to diagonalize this form is again to transform to a spherical representation and consider the invariants $\left[L_{j}^{(n)}\right]$, (cf. equation (19)), rewritten in terms of the spherical basis $\partial Q^{(J)}$ and $\left[Q^{(2)} \otimes \partial Q^{(L)}\right]^{(J)}$. In analogy to the second order invariants, (cf. equation $(8 a)$ ), we can now construct third and fourth order spherical invariants as follows:

$$
\begin{aligned}
& I_{3}(\alpha)=\left[\partial Q^{(J)} \otimes\left[Q^{(2)} \otimes \partial Q^{(L)}\right]^{(J)}\right]^{(0)}, \quad \alpha=1, \ldots, 6 \\
& I_{4}(\beta)=\left[\left[Q^{(2)} \otimes \partial Q^{(L)}\right]^{(J)} \otimes\left[Q^{(2)} \otimes \partial Q^{(M)}\right]^{(J)}\right]^{(0)}, \quad \beta=1, \ldots, 13 .
\end{aligned}
$$

The problem that has to be solved first is the identification of all linearly independent invariants $(20 a, b)$ or equivalently, all relevant representations parametrized by $J, L$ and $M$.

In the search of the invariants the permutation symmetry has to be taken into account. To set the problem more specifically, let us find linearly independent, fourth order, spherical invariants, of the form dictated by equation $(20 \mathrm{~b})$. For this purpose it is convenient to introduce a spherical reference basis of linearly independent invariants, defined as $\left[\left[Q^{(2)} \otimes Q^{(2)}\right]^{\left(l_{12}\right)} \otimes\left[\partial Q^{\left(i_{3}\right)} \otimes \partial Q^{\left(l_{4}\right)}\right]^{\left(l_{12}\right)}\right]^{(0)}\left(l_{3}, l_{4}=1,2,3\right)$. In this basis invariance of the term $\left[Q^{(2)} \otimes Q^{(2)}\right]^{\left(l_{12}\right)}$ with respect to the permutation of $Q^{(2)}$ allows only even values of $l_{12}(=0,2,4)$ where each representation appears exactly once. Now, studying the coupling $\left[\partial Q^{\left(l_{3}\right)} \otimes \partial Q^{\left(l_{4}\right)}\right]^{\left(l_{12}\right)}$, and observing correctly the permutation symmetry with respect to $\partial \mathbf{Q}$, we immediately find that in this tensor product representation $l_{12}=0$ appears three times, representation $l_{12}=2$ appears six times and representation $l_{12}=4$ appears four times. Thus, we again have thirteen linearly independent invariants which can be classified in two groups. The first one contains eight invariants which result from couplings with $l_{3}=l_{4}$ and the second contains five invariants due to couplings with $l_{3} \neq l_{4}$.

Now consider the spherical coupling $(20 b)$. Because the permutation symmetry is not immediately evident, this sort of coupling leads to twenty-three invariants. They are divided into two groups whose members are counted by a simple addition of angular momenta: a group of thirteen positive definite invariants with $L_{3}=M_{4}$ and a group of ten indefinite invariants with $L_{3} \neq M_{4}$. In order to extract thirteen linearly independent terms we expand these twenty-three invariants in our spherical, reference 
basis using $9 j$-symbols [29]

$$
\begin{gathered}
{\left[\left[Q^{(2)} \otimes \partial Q^{(L)}\right]^{(J)} \otimes\left[Q^{(2)} \otimes \partial Q^{(M)}\right]^{(J)}\right]^{(0)}} \\
=\sum_{l} \hat{l} \hat{J}\left\{\begin{array}{ccc}
2 & 2 & l \\
L & M & l \\
J & J & 0
\end{array}\right\}\left[\left[Q^{(2)} \otimes Q^{(2)}\right]^{(l)} \otimes\left[\partial Q^{(L)} \otimes \partial Q^{(M)}\right]^{(0)},\right.
\end{gathered}
$$

where $L, M=1,2,3, \hat{I}=\sqrt{ }(2 I+1)$ and where $\{\ldots\}$ are $9 j$-symbols. The expression $(20 \mathrm{c})$ relates the twenty-three linearly dependent invariants of the type $(20 \mathrm{~b})$ (cf. the left-hand side of equation $(20 c)$ ) to the thirteen linearly independent invariants of our reference basis (cf. the right-hand side of equation $(20 c)$ ) and allows us to extract out of these twenty-three invariants a set of thirteen linearly independent invariants of the form $(20 b)$. As shown in the previous paragraph, these thirteen linearly independent invariants can also be ordered with respect to $L$ and $M$. We find that the case $L=M$ yields eight linearly independent invariants; the remaining five invariants carry $L \neq M$. Relations between $\beta, J, L$ and $M$ are given in table 3 . A similar analysis could be given for the third order invariants, cf. equation $(20 \mathrm{a})$; The results are collected in table 3. The transformation matrices between spherical and cartesian representation are also given in the Appendix.

Table 3. Parameters of the independent invariants of $(a)$ third order, (cf. equation $(20 a)$ ), $(b)$

\begin{tabular}{|c|c|c|c|c|}
\hline & \multicolumn{4}{|c|}{$(a)$} \\
\hline & $\alpha$ & $J$ & $L$ & \\
\hline & 1 & 1 & 1 & \\
\hline & 2 & 2 & 1 & \\
\hline & 3 & 1 & 3 & \\
\hline & 4 & 2 & 2 & \\
\hline & 5 & 2 & 3 & \\
\hline & 6 & 3 & 3 & \\
\hline & \multicolumn{4}{|c|}{ (b) } \\
\hline \multirow{9}{*}{$L=M$} & $\beta$ & $L$ & $M$ & $J$ \\
\hline & 1 & 1 & 1 & 1 \\
\hline & 2 & 1 & 1 & 2 \\
\hline & 3 & 2 & 2 & 0 \\
\hline & 4 & 2 & 2 & 1 \\
\hline & 5 & 2 & 2 & 2 \\
\hline & 6 & 3 & 3 & 1 \\
\hline & 7 & 3 & 3 & 2 \\
\hline & 8 & 3 & 3 & 3 \\
\hline \multirow{5}{*}{$L \neq M$} & 9 & 1 & 2 & 1 \\
\hline & 10 & 1 & 3 & 1 \\
\hline & 11 & 1 & 3 & 2 \\
\hline & 12 & 2 & 3 & 1 \\
\hline & 13 & 2 & 3 & 2 \\
\hline
\end{tabular}
four order, (cf. equation $(20 b)$ ).

After rewriting the expansion (19) in terms of the spherical invariants, (cf. equations $(8 a),(20 a, b)$ and (table 3$))$ we find the alternative expression

$$
\begin{aligned}
F_{\mathrm{N}, \mathrm{el}} & =\sum_{n=1}^{3} K_{n}^{(2)} I_{2}(n)+\sum_{m=1}^{6} K_{m}^{(3)} I_{3}(m)+\sum_{l=1}^{13} K_{l}^{(4)} I_{4}(l) \\
& =\sum_{J=0}^{3} \sum_{\alpha, \beta} C_{\alpha \beta}^{(J)}[T(J, \alpha) \otimes T(J, \beta)]^{(0)},
\end{aligned}
$$


where matrices $C^{(J)}$ are defined as

$$
\begin{aligned}
& C^{(0)}=\left[K_{3}^{(4)}\right], \quad C^{(3)}=\left(\begin{array}{cc}
K_{3}^{(2)} & \frac{1}{2} K_{6}^{(3)} \\
\frac{1}{2} K_{6}^{(3)} & K_{8}^{(4)}
\end{array}\right),
\end{aligned}
$$

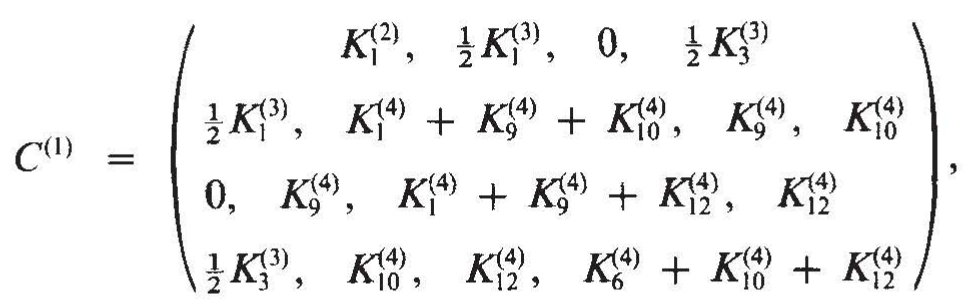

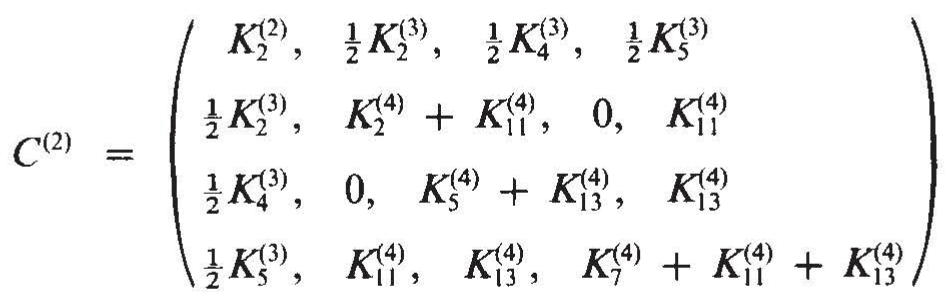

and where

$$
\begin{aligned}
T(0,1) & =\left[Q^{(2)} \otimes \partial Q^{(2)}\right]^{(0)}, \quad T(J, 1)=\partial Q^{(J)}, \quad J=1,2,3, \\
T(J, \gamma+1) & =\left[Q^{(2)} \otimes \partial Q^{(\gamma)}\right]^{(J)}, \quad J=1,2, \quad \gamma=1,2,3, \\
T(3,2) & =\left[Q^{(2)} \otimes \partial Q^{(3)}\right]^{(3)} .
\end{aligned}
$$

Note, that for a given $J, \alpha, T(J, \alpha)$ is the spherical tensor of dimension $2 J+1$. Diagonalization of the form $(20 d)$ leads to

$$
F_{\mathrm{N}, \mathrm{el}}=\sum_{J=0}^{3} \sum_{\alpha} \tilde{K}_{\alpha}^{(J)}[\tilde{T}(J, \alpha) \otimes \tilde{T}(J, \alpha)]^{(0)}
$$

where $\tilde{K}_{\alpha}^{(J)}$ are eigenvalues of the matrices $C^{(J)}$ and where $\tilde{T}(J, \alpha)=0_{\alpha \beta}^{(J)} T(J, \beta)$, here $0^{(J)}$ is the matrix diagonalizing $C^{(J)}$.

It is interesting to note that in equation $(20 e)$ the free energy is expressed as a sum of eleven positive definite spherical invariants $[\tilde{T}(J, \alpha) \otimes \widetilde{T}(J, \alpha)]^{(0)}$. Thus, the formula $(20 e)$ yields directly the stability conditions for the elastic constants $\tilde{K}_{\alpha}^{(J)}$

$$
\tilde{K}_{\alpha}^{(J)} \geqslant 0 .
$$

These are equivalent to the requirement that all main minors of the matrices $C^{(J)}$ be positive. Finally using relations between spherical and cartesian invariants (Appendix) inequalities $(20 f)$ can be rewritten in terms of $L_{n}^{(m)}$. These are sufficient conditions for the free energy density, in equations (19) and $(20 d)$, to be positive definite.

Finally, if contributions to the free energy from the surface terms, (cf. equation $(13 a)-(13 d))$, are negligible in the thermodynamic limit, we can eliminate four invariants from the expansion $(20 d)$, further simplifying the stability conditions.

\section{Oseen-Frank theory and Landau-Ginzburg-de Gennes expansion}

In this section we show how the Frank free energy, which depends on the two independent variables of a unit vector, can be obtained from the free energy expansion in terms of the five independent components of a symmetric, traceless tensor. This question has already been discussed in the literature in the mean field approximation [15-17]. Here we present a systematic approach to the problem. In particular, we study the effect of fluctuations of $\mathbf{Q}$ on the elastic constants. 
The order parameter $\mathbf{Q}(\mathbf{r})$ can, in general, be rewritten in the form

$$
\begin{aligned}
Q_{\alpha \beta}(\mathbf{r})= & S(\mathbf{r})\left[n_{\alpha}(\mathbf{r}) n_{\beta}(\mathbf{r})-\delta_{\alpha \beta} / 3\right]+\zeta(\mathbf{r})\left\{m_{\alpha}(\mathbf{r}) m_{\beta}(\mathbf{r})\right. \\
& \left.-[\hat{n}(\mathbf{r}) \times \hat{m}(\mathbf{r})]_{\alpha} \cdot[\hat{n}(r) \times \hat{m}(r)]_{\beta}\right\},
\end{aligned}
$$

where $n_{\alpha}(\mathbf{r})$ is the direction corresponding to the maximum eigenvalue $\frac{2}{3} S(\mathbf{r})$ of $Q_{\alpha \beta}(\mathbf{r})$, and $m_{\alpha}(\mathbf{r})$, the direction corresponding to the second largest eigenvalue $-\frac{1}{3} S(\mathbf{r})+$ $\zeta(\mathbf{r})$. In the orthonormal coordinate system defined at each point in space by $\hat{n}(\mathbf{r}), \hat{m}(\mathbf{r})$ and $\hat{n}(\mathbf{r}) \times \hat{m}(\mathbf{r})\left(n_{\alpha}(\mathbf{r}) n_{\alpha}(\mathbf{r})=m_{\alpha}(\mathbf{r}) m_{\alpha}(\mathbf{r})=1, n_{\alpha}(\mathbf{r}) m_{\alpha}(\mathbf{r})=0\right), Q_{\alpha \beta}(\mathbf{r})$ has the canonical form of equation (21). For uniaxial nematic liquid crystals with constant $S$ equation (21) reduces to equation (5).

The Landau-de Gennes expansion can be written either in terms of $Q_{\alpha \beta}(\mathbf{r})$ or $[S(\mathbf{r})$, $\left.\zeta(\mathbf{r}), n_{\alpha}(\mathbf{r}), m_{\beta}(\mathbf{r})\right]$. These two expansions must be equivalent order by order. Thus, each term $Q_{\alpha \beta}(\mathbf{r})$ corresponds to exactly one in $\left[S(\mathbf{r}), \ldots, m_{\beta}(\mathbf{r})\right]$. The form of the couplings between $\left[S(\mathbf{r}), \ldots, m_{\beta}(\mathbf{r})\right]$ is dictated by the expansion in $Q_{\alpha \beta}(\mathbf{r})$, because this is the basic order parameter describing the orientational pattern of liquid crystals. It implies, for example, that invariants like $n_{\alpha, \alpha} n_{\beta, \beta}$ only appear coupled to $S^{2}(\mathbf{r})$ or higher powers.

A systematic procedure with which to obtain the OZF free energy from the general expansion in $Q_{\alpha \beta}$ is the following:

(i) First, we substitute the canonical representation, equation (21), into the Landau-Ginzburg-de Gennes free energy and consider the spatial variation of $\hat{n}(\mathbf{r})$ in space as fixed.

(ii) Second, we average the free energy over the remaining fields $\zeta(\mathbf{r}), \hat{m}(\mathbf{r})$ and $\delta S(\mathbf{r})=S(\mathbf{r})-S$ under the constraints $\hat{m}^{2}=1$ and $\hat{n} \cdot \hat{m}=0$. Here $S$ is the mean field value of $S(\mathbf{r})$ in the nematic phase. $S$ can be calculated by minimization of $\mathscr{F}_{m f}$, (cf. equation (26a)) with respect to $\bar{S}\left(\bar{S}=(1 / V) \int_{V} d^{3} \mathbf{r} S(\mathbf{r})\right.$, $\tilde{S}_{\min } \equiv S$ ).

Taking the fields $\delta S(\mathbf{r}), \zeta(\mathbf{r})$ and $\hat{m}(\mathbf{r})$ to be random variables the Frank free energy $\mathscr{F}[\hat{n}(\mathbf{r})]$ can be obtained as a thermal average over all their possible spatial variations

$$
\begin{aligned}
\mathscr{F}[\hat{n}(x)]= & -k T \ln \int_{\hat{m}^{2}(\mathbf{r})=1, \hat{n}(\mathbf{r}) \cdot \hat{m}(\mathbf{r})=0} \mathscr{D}(\zeta(\mathbf{r})) \mathscr{D}(\delta S(\mathbf{r})) \mathscr{D}(\hat{m}(\mathbf{r})) \\
& \times \exp \left\{-\beta \int_{V} d^{3} \mathbf{r} F[\hat{n}(\mathbf{r}), \zeta(\mathbf{r}), \hat{m}(\mathbf{r}), \delta S(\mathbf{r}), \text { derivatives }]\right\},
\end{aligned}
$$

with $\beta^{-1}=k T$, while $\int \mathscr{D}(\zeta(\mathbf{r})) \ldots$ denotes functional integration over all possible fields $\zeta(\mathbf{r}), \ldots[28]$.

For translationally invariant systems the functional integral (22) can be calculated by decomposing $\zeta(\mathbf{r}), \hat{m}(\mathbf{r}), \delta S(\mathbf{r}), \hat{n}(\mathbf{r})$ into Fourier components via

$$
\zeta(\mathbf{r})=\frac{1}{V} \sum_{\mathbf{k}} \zeta_{\mathbf{k}} \exp (-i \mathbf{k} \cdot \mathbf{r})
$$

Because $\zeta(\mathbf{r}) \ldots$ are real fields we have additionally $\zeta_{\mathbf{k}}=\zeta_{-\mathbf{k}}^{*} \ldots$ and the space of the distribution is covered by having the amplitudes $\zeta_{k}, \delta S_{k}$ vary over all complex values, thereby $\mathbf{k}$ is restricted to half space. As long as the volume is finite, the values $\mathbf{k}$ are discrete, and the functions satisfy periodic boundary conditions. This choice guarantees a set of distributions that preserve continuity and differentiability properties. 
The standard coarse graining procedure is introduced by including the Debye cut off for wave vectors

$$
|\mathbf{k}|<\Lambda=\left(6 \pi^{2} \varrho\right)^{1 / 3},
$$

where $\varrho$ is the particle density. The measure is then written as

$$
\left.\begin{array}{l}
D \zeta(\mathbf{r}) \ldots=\prod_{\mathbf{k}>0|\mathbf{k}|<\Lambda} \\
\mathscr{D} \zeta_{\mathbf{k}} \ldots \prod_{\mathbf{k}^{\prime} \neq \mathbf{k}^{\prime \prime}}\left[\delta\left(\hat{m}_{\mathbf{k}^{\prime}}, \hat{m}_{\mathbf{k}^{\prime \prime}}\right) \delta\left(\hat{m}_{\mathbf{k}^{\prime}}, \hat{n}_{\mathbf{k}^{\prime \prime}}\right)\right] \delta\left(\sum_{k} \hat{m}_{\mathbf{k}} \cdot \hat{n}_{-\mathbf{k}}\right) \delta\left(\sum_{\mathbf{k}} \hat{m}_{\mathbf{k}} \cdot \hat{m}_{-\mathbf{k}}-1\right) .
\end{array}\right\}
$$

Assume now that $F$ is given by equation $(4 a)$ with $L_{3}^{(2)}=L_{4}^{(2)}=0$. Substituting equations (21) and (23) into equation (4a) and neglecting the terms that are of order higher than four in fluctuating fields yields $\mathscr{F}[\hat{n}(\mathbf{r})]$ in the form

$$
\mathscr{F}[\hat{n}]=\mathscr{F}_{m f}(S)+\mathscr{F}_{\text {Frank }}+\mathscr{F}_{\text {fluct }},
$$

where

$$
\mathscr{F}_{m f}(\bar{S})=V\left(\frac{2}{3} a\left(T-T^{*}\right) \bar{S}^{2}+\frac{2}{9} B \bar{S}^{3}+\frac{4}{9} C \bar{S}^{4}\right)
$$

is the standard Landau part of the free energy and where

$$
\begin{aligned}
\mathscr{F}_{\text {Frank }}= & \left(2 L_{1}^{(2)}+L_{2}^{(2)}\right) S^{2} \int d^{3} \mathbf{r}\left[(\nabla \cdot n)^{2}+(\hat{n} \times \nabla \times \hat{n})^{2}\right] \\
& +2 L_{1}^{(2)} S^{2} \int d^{3} \mathbf{r}(\hat{n} \times \nabla \times \hat{n})^{2}
\end{aligned}
$$

is the mean field part of OZF free energy [15-17]. The mean field formula $(26 b)$ leads to the well-known degeneracy of the splay and bend elastic constants $K_{11}=K_{33}=2\left(L_{1}^{(2)}+L_{2}^{(2)}\right) S^{2}$. It is perhaps worth mentioning that the parts $\mathscr{F}_{m f}$ and $\mathscr{F}_{\text {Frank }}$ of $\mathscr{F}[\hat{n}]$ could also be obtained in a different way by applying the method of steepest descent [28] to the functional integral, equation (22).

The last part of $\mathscr{F}[\hat{n}]$, denoted as $\mathscr{F}_{\text {fluct }}$ appears as a result of fluctuations of various fields. In order to construct an expansion of $\mathscr{F}_{\text {fluct }}$, we separate the free energy into two parts, a gaussian part $H_{0}$ and a perturbation part $H_{1}$,

$$
\mathscr{F}_{\text {fluct }}=-k T \ln \int \text { (measure, equation (24)) } \exp \left\{\beta\left[H_{0}(\zeta, \partial S)+H_{1}(\zeta, \hat{m}, \partial S, \hat{n})\right]\right\} \text {. }
$$

The gaussian part $H_{0}$ corresponds to the quadratic contribution in the variables $\zeta$ and $\delta S$ to the free energy. All terms higher than quadratic will be kept in $H_{1}$, viewed as perturbation of $H_{0}$. We obtain the following expression for $H_{0}$

$$
H_{0}=\sum_{\substack{\mathbf{k} \\|\mathbf{k}|<\Lambda}}\left[A_{1}\left(\mathbf{k}^{2}\right) \delta S_{\mathbf{k}} \delta S_{-\mathbf{k}}+\frac{2}{3} L_{2}^{(2)} \mathbf{k}^{2} \delta S_{\mathbf{k}} \zeta_{-\mathbf{k}}+A_{2}\left(\mathbf{k}^{2}\right) \zeta_{\mathbf{k}} \zeta_{-\mathbf{k}}\right] \text {, }
$$

with

$$
\begin{aligned}
& A_{1}\left(\mathbf{k}^{2}\right)=\frac{2}{3}\left[A+B S+4 C S^{2}+\left(L_{1}^{(2)}+\frac{1}{6} L_{2}^{(2)}\right) \mathbf{k}^{2}\right] \\
& A_{2}\left(\mathbf{k}^{2}\right)=2\left[A-B S+\frac{4}{3} C S^{2}+\left(L_{1}^{(2)}+\frac{1}{2} L_{2}^{(2)}\right) \mathbf{k}^{2}\right]
\end{aligned}
$$


In $H_{1}$ we neglect all terms proportional to $n_{\alpha} m_{\alpha, \beta}$ that cannot be converted to terms $m_{\alpha} n_{\alpha, \beta}$. This allows us to construct the simplest perturbation scheme that takes into account restrictions on $\hat{m}$ and $\hat{n}$.

In the limit of long wavelength deformations of the director field, $\hat{n}(\mathbf{r})$, the effect of fluctuations on the OZF elastic constants is evaluated by performing a cumulant expansion for $\mathscr{F}_{\text {fluct }}$ about $H_{0}$ with $H_{1}$ as the perturbation. In lowest order we obtain

$$
\begin{aligned}
\mathscr{F}_{\text {fluct }}= & \left\langle H_{1}\right\rangle_{0}+\cdots \\
= & {\left[\left(2 L_{1}^{(2)}+L_{2}^{(2)}\right)\left(\left\langle\delta S^{2}\right\rangle_{0}-\left\langle\zeta^{2}\right\rangle_{0}\right)+\frac{1}{4} L_{2}^{(2)}\left\langle\zeta^{2}\right\rangle_{0}\right] } \\
& \times \int(\nabla \cdot \hat{n})^{2} d^{3} \mathbf{r}+\left[2 L_{1}^{(2)}\left(\left\langle\delta S^{2}\right\rangle_{0}-\left\langle\zeta^{2}\right\rangle_{0}\right)-\frac{3}{4} L_{2}^{(2)}\left\langle\zeta^{2}\right\rangle_{0}\right] \\
& \times \int(\hat{n} \cdot \nabla \times \hat{n})^{2} d^{3} \mathbf{r}+\left[2 L_{1}^{(2)}\left(\left\langle\delta S^{2}\right\rangle_{0}-\left\langle\zeta^{2}\right\rangle_{0}\right)\right. \\
& \left.+L_{2}^{(2)}\left(\left\langle\delta S^{2}\right\rangle_{0}+2\langle\delta S \zeta\rangle_{0}\right)\right] \int(\hat{n} \times \nabla \times \hat{n})^{2} d^{3} \mathbf{r}+\cdots,
\end{aligned}
$$

where

$$
\langle A\rangle_{0}=Z_{0}^{-1} \int \text { (measure, equation }(24) A(\zeta, \delta S, \ldots, \hat{n}) \exp \left(-\beta H_{0}\right)
$$

and

$$
Z_{0}=\langle 1\rangle_{0}
$$

Explicit calculation yields

$$
\begin{aligned}
\left\langle\delta S^{2}\right\rangle_{0} & =\beta^{-1} \int_{0}^{\Lambda} \frac{k^{2} d k}{4 \pi^{2}} \frac{A_{1}\left(k^{2}\right)}{\operatorname{det} A_{0}}, \\
\left\langle\zeta^{2}\right\rangle_{0} & =\beta^{-1} \int_{0}^{\Lambda} \frac{k^{2} d k}{4 \pi^{2}} \frac{A_{2}\left(k^{2}\right)}{\operatorname{det} A_{0}}, \\
\langle\delta S \zeta\rangle_{0} & =-\beta^{-1} \int_{0}^{\Lambda} \frac{k^{2} d k}{4 \pi^{2}}\left(\frac{1}{3} L_{2}^{(2)} \mathbf{k}^{2}\right)\left[\operatorname{det} A_{0}\right]^{-1},
\end{aligned}
$$

where

$$
A_{0}=\left[\begin{array}{cc}
A_{1}\left(k^{2}\right), & \frac{1}{3} L_{2}^{(2)} k^{2} \\
\frac{1}{3} L_{2}^{(2)} k^{2}, & A_{2}\left(k^{2}\right)
\end{array}\right] .
$$

A more comprehensive analysis will be given in a forthcoming publication [30]. The main results can be summarized as follows.

In general, the effect of fluctuations in the nematic phase is to renormalize the Frank elastic constants. In the approximation that takes into account only quadratic terms in the fluctuating fields this renormalization does not remove the degeneracy of $K_{11}$ and $K_{33}$. However, it leads to a non-standard temperature dependence of the elastic constants, keeping them non-zero even for $S=0$. Interestingly, the higher 
Table 4. Mean field decomposition of $\partial \mathbf{Q} \partial \mathbf{Q}, \mathbf{Q} \partial \mathbf{Q} \partial \mathbf{Q}$ and $\mathbf{Q} \mathbf{Q} \partial \mathbf{Q} \partial \mathbf{Q}$ invariants onto splay, twist, bend and surface components.

\begin{tabular}{lcccc}
\hline Invariant & $(\nabla \cdot \hat{n})^{2}$ & $(\hat{n} \cdot \nabla \times \hat{n})^{2}$ & $(\hat{n} \times \nabla \times \hat{n})^{2}$ & $n_{i, j} n_{j, i}-n_{i, i} n_{j, j}$ \\
\hline$\left[L_{1}^{(2)}\right] / S^{2}$ & 2 & 2 & 2 & 2 \\
{$\left[L_{2}^{(2)}\right] / S^{2}$} & 1 & 0 & 1 & 0 \\
{$\left[L_{3}^{(2)}\right] / S^{2}$} & 1 & 0 & 1 & 1 \\
{$\left[L_{3}^{(3)}\right] / S^{3}$} & 0 & 0 & 0 & 0 \\
{$\left[L_{2}^{(3)}\right] / S^{3}$} & $-1 / 3$ & 0 & $2 / 3$ & 0 \\
{$\left[L_{3}^{(3)}\right] / S^{3}$} & $2 / 3$ & 0 & $-1 / 3$ & 0 \\
{$\left[L_{4}^{(3)}\right] / S^{3}$} & $1 / 3$ & $1 / 3$ & $1 / 3$ & $1 / 3$ \\
{$\left[L_{5}^{(3)}\right] / S^{3}$} & $2 / 3$ & 0 & $-1 / 3$ & $2 / 3$ \\
{$\left[L_{6}^{(3)}\right] / S^{3}$} & $-1 / 3$ & 0 & $2 / 3$ & $-1 / 3$ \\
{$\left[L_{(4)}^{(4)}\right] / S^{4}$} & $2 / 3$ & 0 & $2 / 3$ & 0 \\
{$\left[L_{2}^{(4)}\right] / S^{4}$} & $4 / 3$ & $4 / 3$ & $4 / 3$ & $4 / 3$ \\
{$\left[L_{3}^{(3)}\right] / S^{4}$} & $2 / 3$ & 0 & $2 / 3$ & $2 / 3$ \\
{$\left[L_{4}^{(4)}\right] / S^{4}$} & 0 & 0 & 0 & 0 \\
{$\left[L_{5}^{(4)}\right] / S^{4}$} & $1 / 9$ & 0 & $4 / 9$ & 0 \\
{$\left[L_{6}^{(4)}\right] / S^{4}$} & $4 / 9$ & 0 & $1 / 9$ & 0 \\
{$\left[L_{7}^{(4)}\right] / S^{4}$} & $5 / 9$ & $5 / 9$ & $5 / 9$ & $5 / 9$ \\
{$\left[L_{8}^{(4)}\right] / S^{4}$} & $4 / 9$ & 0 & $1 / 9$ & $4 / 9$ \\
{$\left[L_{9}^{(4)}\right] / S^{4}$} & $1 / 9$ & 0 & $4 / 9$ & $1 / 9$ \\
{$\left[L_{10}^{(4)}\right] / S^{4}$} & $-2 / 9$ & 0 & $-2 / 9$ & 0 \\
{$\left[L_{11}^{(4)}\right] / S^{4}$} & $-1 / 9$ & 0 & $2 / 9$ & $-1 / 9$ \\
{$\left[L_{12}^{(4)}\right] / S^{4}$} & 0 & 0 & 0 & 0 \\
{$\left[L_{13}^{(4)}\right] / S^{4}$} & 0 & 0 & 0 & 0 \\
\hline
\end{tabular}

order terms, proportional to $m_{\alpha} m_{\beta}$ remove the degeneracy of $K_{11}$ and $K_{33}$

$$
K_{33}-K_{11}=L_{2}^{(2)}\left(\frac{3}{4}\left\langle\zeta^{2}\right\rangle_{0}+2\langle\delta S \zeta\rangle_{0}\right)>0
$$

where the last inequality is valid provided that $L_{1}^{(2)}$ and $L_{2}^{(2)}$ fulfil the stability conditions in equation (19) with $L_{3}^{(2)}=0$.

In still higher order than presently treated we observe a non-local interaction between the director field and its derivatives like $\int d^{3} \mathbf{r} d^{3} \mathbf{r}^{\prime} K\left(\mathbf{r}, \mathbf{r}^{\prime}\right)\left[\partial_{\alpha} n_{\alpha}(\mathbf{r})\right]^{2}\left[\partial_{\alpha} n_{\alpha}\left(\mathbf{r}^{\prime}\right)\right]^{2}$, where the kernel $K\left(\mathbf{r}, \mathbf{r}^{\prime}\right) \equiv K\left(\mathbf{r}-\mathbf{r}^{\prime}\right)$ is nearly the Fourier transform to real space of the mode-mode correlation functions.

Inclusion of higher order couplings, like $\mathbf{Q} \partial \mathbf{Q} \partial \mathbf{Q}$ and $\mathbf{Q} \mathbf{Q} \partial \mathbf{Q} \partial \mathbf{Q}$ to the free energy expansion, makes the reduction to the Oseen-Frank description rather difficult. For that reason we restrict the analysis of higher order tensor couplings to the presentation of mean field results. These are collected in table 4 . Note that our expressions for $L_{m}^{(3)}$ are different from those given by Berreman and Meiboom (BM) [17]. In their expression $G_{4}^{(3)}$ (equivalent to our $\left[L_{5}^{(3)}\right]$ ) there is a contribution from the twist deformation, which in our expression is replaced by surface term. Additionally, in the BM formulae the factor $1 / 3$ is systematically missing. From table 4 it follows that there are five different third order and ten fourth order expressions which are not identically zero for uniaxial nematics with a position independent order parameter $S$. In third order we have one relation less than BM. Since there are more tensor functions, $\left[L_{n}^{(m)}\right]$, than OZF elastic terms, the tensor functions are not all independent for strictly uniaxial variations of $\mathbf{Q}$ with constant $S$. These relations can be found with 
the results of table 4

$$
\begin{aligned}
{\left[L_{1}^{(3)}\right] } & =0, \\
2\left[L_{2}^{(3)}\right]+\left[L_{3}^{(3)}\right]-\left[L_{5}^{(3)}\right]-2\left[L_{6}^{(3)}\right] & =0, \\
5\left[L_{1}^{(4)}\right]-6\left[L_{5}^{(4)}\right]-6\left[L_{6}^{(4)}\right] & =0, \\
5\left[L_{2}^{(4)}\right]-12\left[L_{7}^{(4)}\right] & =0, \\
{\left[L_{1}^{(4)}\right]+4\left[L_{3}^{(4)}\right]-6\left[L_{5}^{(4)}\right]-6\left[L_{8}^{(4)}\right] } & =0, \\
{\left[L_{1}^{(4)}\right]-\left[L_{3}^{(4)}\right]-6\left[L_{5}^{(4)}\right]+6\left[L_{9}^{(4)}\right] } & =0, \\
{\left[L_{1}^{(4)}\right]+3\left[L_{10}^{(4)}\right] } & =0, \\
2\left[L_{1}^{(4)}\right]+\left[L_{2}^{(4)}\right]-12\left[L_{5}^{(4)}\right]+12\left[L_{11}^{(4)}\right] & =0, \\
{\left[L_{4}^{(4)}\right]=\left[L_{12}^{(4)}\right]=\left[L_{13}^{(4)}\right] } & =0 .
\end{aligned}
$$

Berreman and Meiboom [17] give two rather than one relation among the third order expressions. The second one, however, is not independent because of the general relation $(13 f)$. To complete this paragraph we give mean field formulae for the cholesteric pitch. Up to fourth order in $S$ we find from equation (1) and (14)

$$
q_{0} K_{22} \simeq S^{2} L_{4}^{(2)}-S^{3} \frac{L_{7}^{(3)}}{3}-\frac{2}{3} S^{4}\left(\frac{1}{3} L_{14}^{(4)}-L_{15}^{(4)}\right)
$$

\section{Generalized Landau-de Gennes expansion versus Meyer theory}

As we have discussed in the Introduction Meyer [19] was the first to point out that the polarization density field, $\mathbf{P}(\mathbf{r})$, could play an important role in the continuum theory of liquid crystals. He showed that for nematics the presence of $\mathbf{P}$ leads to new elastic terms, $F_{p}$, that have to be added to the OZF expression (1). Including only lowest order interactions between the different fields he showed that

$$
\begin{aligned}
F_{p}= & \frac{1}{2} \alpha_{1} P_{\|}^{2}+\frac{1}{2} \alpha_{3} P_{\perp}^{2}-\frac{1}{8 \pi} \varepsilon_{\|}^{0} E_{\|}^{2}-\frac{1}{8 \pi} \varepsilon_{\perp}^{0} E_{\perp}^{2} \\
& -e_{1}^{0} E_{\|}(\boldsymbol{\nabla} \cdot \hat{n})-e_{3}^{0} \mathbf{E}_{\perp} \cdot(\hat{n} \times \nabla \times \hat{n}) \\
& -a_{1} P_{\|}(\boldsymbol{\nabla} \cdot \hat{n})-a_{3} \mathbf{P}_{\perp} \cdot(\hat{n} \times \nabla \times \hat{n}) \\
& -\mu_{1} P_{\|} E_{\|}-\mu_{3} \mathbf{P}_{\perp} \cdot \mathbf{E}_{\perp},
\end{aligned}
$$

where $\|(\perp)$ denotes component, or vector parallel (perpendicular) to $\hat{n}$. The coefficients $\alpha_{\perp}, \ldots, \mu_{3}$ are new elastic constants.

The most interesting terms identified by Meyer are those weighted by the flexoelectric coefficients $e_{1}^{0}$ and $e_{3}^{0}$ and the flexopolarization coefficients $a_{1}$ and $a_{3}$. They describe how the basic deformations of the director field couple to the polarization and an external electric field. Because flexoelectricity remains one of the few fundamental effects in liquid crystal physics which were predicted primarily by symmetry arguments, it appears interesting to establish the equivalent tensor invariants. This again is easily achieved by applying the mean field approximation to the results of $\S 3$. In particular, substitution of equation (5) into equations $(15 a)-(15 d)$ yields

$$
\left.\begin{array}{l}
a_{1}=-S A_{1}^{(2)}-S^{2}\left(\frac{2}{3} A_{1}^{(3)}-\frac{1}{3} A_{3}^{(3)}\right)+S^{3}\left(\frac{2}{9} A_{2}^{(4)}-\frac{4}{9} A_{4}^{(4)}-\frac{2}{3} A_{5}^{(4)}\right), \\
a_{3}=S A_{1}^{(2)}-S^{2}\left(\frac{1}{3} A_{1}^{(3)}-\frac{2}{3} A_{3}^{(3)}\right)-S^{3}\left(\frac{2}{9} A_{2}^{(4)}+\frac{1}{9} A_{4}^{(4)}+\frac{2}{3} A_{5}^{(4)}\right),
\end{array}\right\}
$$


similar relations hold for the coefficients $e_{1}^{0}$ and $e_{3}^{0}$. From expressions (36) it follows that the coefficients $a_{1}, a_{3}$ (or $e_{1}^{0}, e_{3}^{0}$ ) are related. For instance, up an order linear in $S$ they should only differ in sign. If chiral terms are included, we have to add cross-coupling terms of the form

$$
\left.\begin{array}{l}
{\left[\zeta_{3}^{(3)}\right]=-S(\mathbf{E} \cdot \hat{n}) \mathbf{P} \cdot(\boldsymbol{\nabla} \times \hat{n})-S \hat{n} \cdot[\mathbf{P} \times \boldsymbol{\nabla}(\mathbf{E} \cdot \hat{n})],} \\
{\left[\zeta_{4}^{(3)}\right]=S(\boldsymbol{\nabla} \cdot \hat{n}) \mathbf{E} \cdot(\hat{n} \times \mathbf{P})-S \mathbf{E} \cdot[(\hat{n} \times \nabla \times \hat{n}) \times \mathbf{P}] .}
\end{array}\right\}
$$

Again, we would like to emphasize that the relations (36) and (37) apply only as long as the nematic liquid crystal is strictly uniaxial.

\section{Relations between $L_{n}^{(m)}$ and the OZF elastic constants}

We have shown how the Landau-Ginzburg-de Gennes expansion for the free energy of a strictly uniaxial strained nematic liquid crystal is related to well-known OZF expansion. The simplest relations are obtained in the mean field approximation (see table 4 and equation (33)). As these equations also relate the OZF elastic constants $K_{i j}$ with the Landau-Ginzburg-de Gennes elastic constants $L_{n}^{(m)}$, it is possible to estimate values for some of the $L_{n}^{(m)}$ from existing experimental data on the temperature dependence of $K_{i j}$. From table 4 it follows immediately that

$$
\frac{K_{i j}}{S^{2}}=K_{i j}^{(2)}+S K_{i j}^{(3)}+S^{2} K_{i j}^{(4)}, \quad(i j)=(11),(22),(33),(24),
$$

where $K_{i j}^{(m)}$ are linear functions of $L_{n}^{(m)}(m=2,3,4)$ and where $K_{11}^{(2)}=K_{33}^{(2)}$. This result generalizes to the case of higher order invariants of the form $\mathbf{Q} \ldots \mathbf{Q} \partial \mathbf{Q} \partial \mathbf{Q}$. Summing up the terms in orders of $S$ we arrive at $K_{i j}=S^{2} f_{i j}(S)$, where $f_{i j}(S)$ is, in principle, an arbitrary function of $S$.

As there are more elastic constants $L_{n}^{(m)}$ than $K_{i j}^{(m)}$, it is not possible to find a one-to-one correspondence between them without resorting to some additional approximations or complementary information. In principle two different solutions to this problem are immediately clear. First, we assume that the tensor field $\mathbf{Q}(r)$ is known and that surface energies, (cf. equations. $(13 a)-(13 d)$ ) of $\mathbf{Q}$ are negligible in the thermodynamic limit. With this assumption we can eliminate four elastic terms, say $\left[L_{3}^{(2)}\right],\left[L_{6}^{(3)}\right],\left[L_{10}^{(4)}\right]$ and $\left[L_{9}^{(4)}\right]$, from the general expansion in $\left[L_{m}^{(n)}\right]$. Now, using the decomposition of $\mathbf{Q}(\mathbf{r})$ in equation (21), we calculate the $\hat{n}(\mathbf{r})$ contribution to the elastic free energy. Comparing it with the OZF free energy gives

$$
\begin{aligned}
L_{1}^{(2)} & =\frac{1}{4} K_{22}^{(2)}, \\
L_{2}^{(2)} & =\frac{1}{2}\left(K_{11}^{(2)}-K_{22}^{(2)}\right), \\
L_{4}^{(3)} & =\frac{3}{2} K_{22}^{(3)}, \\
L_{2}^{(3)} & =\frac{1}{2}\left(K_{11}^{(3)}+2 K_{33}^{(3)}-3 K_{22}^{(3)}\right), \\
L_{3}^{(3)}+L_{5}^{(3)} & =K_{11}-\frac{3}{2} K_{22}+\frac{1}{2} K_{33}, \\
12 L_{2}^{(4)}+5 L_{7}^{(4)}-L_{11}^{(4)} & =\frac{9}{2} K_{22}^{(4)}, \\
-L_{5}^{(4)}+L_{6}^{(4)}+L_{8}^{(4)}-L_{11}^{(4)} & =\frac{3}{2}\left(K_{11}^{(4)}-K_{33}^{(4)}\right), \\
6\left(L_{1}^{(4)}+L_{3}^{(4)}\right)+5 L_{5}^{(4)}+4 L_{11}^{(4)} & =\frac{2}{3}\left(4 K_{33}^{(4)}-K_{11}^{(4)}-3 K_{22}^{(4)}\right) .
\end{aligned}
$$


As we can see from equation (39), only a few elastic constants are estimated using this method. The remaining ones appear in linear combinations and nine complementary equations are needed relating these elastic constants with elastic constants of the field $\hat{m}$ to find one-to-one relations. The estimated values could, in principle, be tested against the stability conditions given in $\$ 4$ but because of the high number of free parameters, these inequalities are not very restrictive.

An alternative approach is to start from a given director field, $\hat{n}(\mathbf{r})$. Using equation (5) we now construct a uniaxial approximation for $Q_{\alpha \beta}$. In this case invariants $\left[L_{m}^{(n)}\right]$ are no longer independent and the uniaxial restrictions, (cf. equation (33)), have to be taken into account. In addition surface energies cannot be disregarded.

Neglecting invariants $\left[L_{m}^{(n)}\right]$ with high index $m$ (as dictated by relations (33)) and resorting to the assumption of Nehring and Saupe, (cf. equation $(3 a)$ ) it follows that

$$
\begin{aligned}
& L_{1}^{(2)}=\frac{1}{4} K_{22}^{(2)}, \quad L_{2}^{(2)}=L_{3}^{(2)}=\frac{1}{4}\left(K_{11}^{(2)}-K_{22}^{(2)}\right), \\
& L_{2}^{(3)}=K_{33}^{(3)}-\frac{3}{2} K_{22}^{(3)}+\frac{1}{2} K_{11}^{(3)}, \\
& L_{3}^{(3)}=\frac{1}{2} K_{33}^{(3)}-\frac{9}{8} K_{22}^{(3)}+\frac{5}{8} K_{11}^{(3)}, \quad L_{4}^{(3)}=\frac{3 K_{22}^{(3)}}{2}, \\
& L_{5}^{(3)}=\frac{3}{8}\left(K_{11}^{(3)}-K_{22}^{(3)}\right), \\
& L_{1}^{(4)}=-\frac{1}{4} K_{33}^{(4)}-\frac{3}{8} K_{22}^{(4)}+\frac{5}{8} K_{11}^{(4)}, \quad L_{2}^{(4)}=\frac{3}{8} K_{22}^{(4)}, \\
& L_{3}^{(4)}=\frac{3}{8}\left(K_{11}^{(4)}-K_{22}^{(4)}\right), \quad L_{5}^{(4)}=\frac{3}{2}\left(K_{33}^{(4)}-K_{11}^{(4)}\right) .
\end{aligned}
$$

Taking the experimental data for 4-methoxybenzylidine-4'- $n$-butylaniline (MBBA) and E7 from [10,11,17], using relations (33), table 3 and resorting to the approximation $(3 a)$, we obtain values for $K_{i i}^{(n)}$ as listed in table 5 . These parameters are imprecise as they are strongly based on the assumption, (cf. equation (5)) of uniaxiality. We believe, however, that they are good enough to provide some insight into the importance of higher order elastic terms. They are also different from those of Berreman and Meiboom [17]. The stability relations allow us additionally to set inequalities for the elastic constants that are not present in the uniaxial approximation.

Table 5. Elastic constants $K_{i i}^{(n)}, n=2,3,4$ for two nematogens.

\begin{tabular}{lrr}
\hline & E7 & MBBA \\
\hline$K_{1}^{(2)}$ & 3.69 & 1.53 \\
$K_{1}^{(3)}$ & -6.46 & -1.08 \\
$K_{1}^{(4)}$ & $5 \cdot 51$ & 1.03 \\
$K_{2}^{(2)}$ & 2.90 & 1.27 \\
$K_{2}^{(3)}$ & -5.04 & -1.56 \\
$K_{2}^{(4)}$ & 4.14 & 1.32 \\
$K_{3}^{(3)}$ & -6.21 & -0.55 \\
$K_{3}^{(4)}$ & 7.49 & 1.07 \\
\hline
\end{tabular}

The elastic constants are given in $10^{-6} \mathrm{dyn}$ and the estimated error $\left|\Delta K_{i i}\right| \lesssim 0.05$ for $i=1,2,3$.

\section{Summary}

In this article we have established a Landau-Ginzburg-de Gennes expansion for the orientational part of the free energy of nematic and cholesteric liquid crystals, 
which is complete up to fourth order in the order parameter $\mathbf{Q}$ and its gradient $\partial \mathbf{Q}$. In addition, the expansion includes couplings of the order parameter with external electric and magnetic fields $\mathbf{E}, \mathbf{H}$ and a polarization field $\mathbf{P}$.

Special cases of second and third order expansions have already been proposed in the literature $\{16,17,18,26]$. However, they failed to recognize the minimal set of independent invariants in third order of the orientation tensor $\mathbf{Q}$. More importantly, the results of these studies apply on to strictly uniaxial phases with a position independent order parameter $S$. When biaxiality is taken into account these free energies appear to be thermodynamically unstable. In $[17,18,26]$ the number of independent invariants for the general, biaxial case was overestimated because a cartesian representation has been used, which cannot easily incorporate the condition that tensor order parameter $\mathbf{Q}$ and its derivatives be traceless. Thus, an alternative approach with which to construct the elastic free energy has been formulated based upon the spherical tensor analysis. All invariants have been found employing the spherical representation for $\mathbf{Q}, \partial \mathbf{Q}, \mathbf{P}, \partial \mathbf{P}, \mathbf{E}$ and $\mathbf{H}$. Various final forms of this expansion have been written in both the spherical and cartesian representations. In the presentation all terms have also been classified with respect to space-time reversal symmetries.

The analysis presented is more rigorous than previous studies in that the number, the explicit form and the linear independence of invariants is immediately evident. The method allows us to find all corresponding linearly independent surface terms. For many applications, for example, for the normal modes of the hydrodynamic equations the surface contributions do not play a role. The topological structure of $\mathbf{Q}$ suggests that these contributions should also be negligible in the elastic theory. In all these cases, surface relations reduce the number of independent invariants and simplify the theory. It is perhaps worth mentioning that the problem of finding all surface relations for higher orders is non-trivial.

Let us now concentrate on some specific results.

It has been emphasized that for general, biaxial nematics with $\mathbf{P}=\mathbf{E}=\mathbf{H}=0$ the correct expansion, that takes into account second and third order terms $\partial \mathbf{Q} \partial \mathbf{Q}$ and $\mathbf{Q} \partial \mathbf{Q} \partial \mathbf{Q}$, respectively, has to be completed by fourth order terms $\mathbf{Q} \mathbf{Q} \partial \mathbf{Q} \partial \mathbf{Q}$. This expansion counts, in general, twenty-two invariants and four surface relations.

With the help of the spherical representation, the general expansion with all twentytwo terms has been rewritten as a sum of only eleven positive definite spherical invariants, (cf. equations $(20 d, e)$ ) leading to simple stability restrictions on the elastic constants.

The expansion as given by equations $(20 d, e)$ can be easily generalized to the case of cholesterics. We note that to include the lowest order cholesteric term $\left[L_{4}^{(2)}\right]$, it is sufficient to replace the invariant $\left[Q^{(2)} \partial Q^{(2)}\right]^{(0)}\left[Q^{(2)} \partial Q^{(2)}\right]^{(0)}$ by $\left[\left[Q^{(2)} \partial Q^{(2)}\right]^{(0)}-\lambda\right]$ $\left[\left[Q^{(2)} \partial Q^{(2)}\right]^{(0)}-\lambda\right]$ where $\lambda$ is a parameter.

At this point one might ask if it is at all necessary to include in the free energy expansion terms that are higher than quadratic in $\partial \mathbf{Q}$. To answer this question, we just mention as an example that in the proper description of the cholesteric blue phase both the higher elastic and chiral terms may play a dominant role for these phases to exist at all. Structures that favour bulk terms in the free energy do not take full advantage of possibilities for minimizing the elastic energy, and vice versa. This difficulty leads to compromise textures (see, for example, [27]). Blue phases exist only in an extremely small temperature range and thus they must be very sensitive even to minor changes in the values of elastic constants. Thus, addition of new terms may cause considerable changes to the equilibrium solutions for $\mathbf{Q}$ and for the value of the cholesteric pitch. 
The phase transitions to nematic, cholesteric or blue phases are strongly first order. Just below the isotropic transition the order parameter, $S$, is usually more than 40 per cent of its maximal possible value. Thus, the elastic terms $\mathbf{Q} \partial \mathbf{Q} \partial \mathbf{Q}$ and $\mathbf{Q} \mathbf{Q} \partial \mathbf{Q} \partial \mathbf{Q}$ are of the same order as the bulk terms $\operatorname{Tr} \mathbf{Q}^{3}$ and $\left(\operatorname{Tr} \mathbf{Q}^{2}\right)^{2}$, respectively. The latter are normally included in the free energy expansion. Finally, the restriction that the tensor $\mathbf{Q}$ be uniaxial is generally incorrect in the neighbourhood of defects [31] and thus it is not sufficient in the theory of blue phases to consider only part of the third order terms. A stable expansion requires also higher, fourth order terms and all approximations must be consistent with the stability requirements. For this purpose, the spherical form, (cf. equation $(20 e)$ ) seems to be especially useful. Using equation $(20 \mathrm{e})$ all approximations can be performed in accord with the requirement of stability.

For those who prefer to work in cartesian representation we have presented explicit relations between the spherical and the cartesian invariants.

A second, new aspect which the present theory accounts for is the polarization field. For many years the investigation of the ferroelectricity in liquid crystals has been restricted to the study of induced effects $[20,22]$. Only recently has evidence become available that liquid crystals consisting of molecules with strongly polar end groups (cyano or nitro) give rise to spontaneous polar states [21], that are directly related to antiferroelectric interactions between molecules.

In general, the electric polarization can be considered to consist of four parts:

(a) The polarization induced by an electric field due to the dielectric polarizability of the material.

(b) The deformation-induced polarization which at each point depends on $\mathbf{Q}$ and $\partial \mathbf{Q}$.

(c) The polarization associated with the gradient of $S$.

(d) Spontaneous polarization due to the presence of permanent dipole moments.

Current theories are concerned only with some special aspects of mechanisms $(b)$ and $(c)$. Our expressions include cases $(b),(c)$ and $(d)$. The first part of the polarization cannot be expressed in terms of local qualities. It depends on the polarization in the whole sample.

Again cholesteric blue phases could be one example for which this additional order parameter is important (see, for example, [2]). It is quite possible that the core of disclination lines and of point defects also displays local electric order. The existence of several new chiral cross-coupling terms between $\mathbf{E}, \mathbf{P}$ and $\mathbf{Q}$, (cf. equation (17)) could be of importance to our understanding of blue phases. Note, that these terms are absent when only a magnetic field is switched on. This magneticelectric field symmetry of lowest order couplings should be seen experimentally for strongly polar compounds. Probably some of the experimental results of Stegemeyer et al. [2] can be accounted for by including some of these new terms.

In $\$ \S 5$ and 6 we proposed a general method of relating the OZF theory, the Meyer theory and the general Landau-Ginzburg-de Gennes expansion. Inherent in the method is the assumption that the main contribution to the elastic free energy comes from the long range director deformations and that biaxiality is only a short range effect due to conformational changes at the molecular level. It is clear that with this assumption the free energy of the director field can be calculated as a thermodynamic average over biaxial degrees of freedom for fixed $\hat{n}(\mathbf{r})$ and $S$. 
It has been demonstrated that the main effect of the fluctuations is to introduce a non-linear dependence of the OZF elastic constants on the bare parameters of the Landau-Ginzburg-de Gennes expansion. More profoundly, elastic and bulk properties couple in such a generalized theory. In addition, the results of previous publications [16-19] can be recovered as special, mean field cases of our general treatment.

Our calculations allow us to draw further conclusions concerning the temperature dependence of the elastic constants. In particular, it has been shown that biaxial fluctuations remove the mean field degeneracy of $K_{11}$ and $K_{33}$ without the necessity of introducing new elastic constants. In the approximation that neglects derivatives of $\hat{m}$ we find $K_{33}>K_{11}$. These results are in agreement with experiments in ordinary nematics $[10,12]$ and provide an alternative solution of the problem of degeneracy, $K_{11}=K_{33}$, to those proposed by Rościszewski [19] and Schiele and Trimper [16].

Section 5 and 6 are completed with the list of mean field relations between the various theories. Finally, generalizing the analysis of Berreman and Meiboom [17], some numerical estimates have been made of the tensor field elastic constants using mean field relations and experimental data for the OZF elastic constants. The complete set of values could, however, be obtained only after finding a way to measure complementary elastic constants of the biaxial field.

All the tedious calculations necessary to obtain the formulas presented in the paper were performed using MACSYMA and SCHOONSCHIP computer programs, which are specially designed for algebraic manipulations.

We hope that this work supplies a useful framework for some more detailed considerations of liquid crystal structures.

\section{Appendix}

In this Appendix we use relations between spherical and cartesian representations to prove linear independence of choosen set of invariants $\mathbf{Q} \partial \mathbf{Q} \partial \mathbf{Q}$ and $\mathbf{Q} \mathbf{Q} \partial \mathbf{Q} \partial \mathbf{Q}$. For completeness we also list the formulas for $\partial \mathbf{Q} \partial \mathbf{Q}$. First, the set of linearly independent spherical invariants is given. Next, these invariants are expanded in terms of cartesian ones. The existence of inverse transformation proves their linear independence. The formulas below are also useful in finding stability conditions in various representations. Let us define

$$
\text { (a) } \begin{aligned}
\tilde{I}_{2}(1) & =\frac{5}{\sqrt{3}} I_{2}(1), \quad \hat{I}_{2}(2)=\frac{15}{\sqrt{5}} I_{2}(2), \\
\hat{I}_{2}(3) & =\frac{105}{\sqrt{ } 7} I_{2}(3)
\end{aligned}
$$

where $I_{2}(L)$ are given by equation $(8 a)$.

$$
\begin{aligned}
\text { (b) } \quad \tilde{I}_{3}(1) & =\frac{5 \sqrt{ } 5}{3} I_{3}(1), \quad \tilde{I}_{3}(2)=\frac{15}{\sqrt{3}} I_{3}(2), \\
\tilde{I}_{3}(3) & =5 \sqrt{105} I_{3}(3), \quad \tilde{I}_{3}(4)=\frac{1}{\sqrt{105}} I_{3}(4), \\
\tilde{I}_{3}(5) & =\frac{15 \sqrt{ } 14}{\sqrt{3}} I_{3}(5), \quad \tilde{I}_{3}(6)=\frac{525}{\sqrt{70}} I_{3}(6), \\
\text { (c) } \tilde{I}_{4}(1) & =\frac{25 \sqrt{ } 3}{9} I^{4}(1), \quad \tilde{I}_{4}(2)=5 \sqrt{ } 5 I_{4}(2),
\end{aligned}
$$




$$
\begin{aligned}
\tilde{I}_{4}(3) & =\frac{15}{2} I_{4}(3), \quad \tilde{I}_{4}=15 \sqrt{ } 3 I_{4}(4), \\
\tilde{I}_{4}(5) & =21 \sqrt{ } 5 I_{4}(5), \quad \tilde{I}_{4}(6)=525 \sqrt{ } 3 I_{4}(6), \\
\tilde{I}_{4}(7) & =210 \sqrt{ } 5 I_{4}(7), \quad \tilde{I}_{4}(8)=450 \sqrt{ } 7 I_{4}(8), \\
\tilde{I}_{4}(9) & =5 \sqrt{ } 5 I_{4}(9), \quad \tilde{I}_{4}(10)=25 \sqrt{ } 7 I_{4}(10), \\
\tilde{I}_{4}(11) & =5 \sqrt{ } 70 I_{4}(11), \quad \tilde{I}_{4}(12)=15 \sqrt{ } 105 I_{4}(12), \\
\tilde{I}_{4}(13) & =105 \sqrt{ } 2 I_{4}(13) .
\end{aligned}
$$

Now, expressing $\tilde{I}_{n}(i)$ as linear combinations of $\left[L_{i}^{(n)}\right]$

$$
\tilde{I}_{n}(i)=\left(A^{(n)}\right) i j\left[L_{i}^{(n)}\right] \text {, }
$$

where $n=2,3,4, i=1, \ldots, \alpha_{n}, \alpha_{2}=3, \alpha_{3}=6, \alpha_{4}=13$, one finds

$$
\begin{aligned}
& A^{(2)}=\left[\begin{array}{rrr}
0 & 1 & 0 \\
2 & -1 & -2 \\
5 & -4 & 10
\end{array}\right] \text {; } \\
& A^{(3)}=\left[\begin{array}{rrrrrr}
0 & 0 & 1 & 0 & 0 & 0 \\
-2 & 2 & 1 & 0 & 0 & 0 \\
5 & 10 & -4 & 0 & 0 & 0 \\
4 & -4 & -1 & 4 & -4 & 0 \\
7 & -2 & -6 & 10 & 0 & -10 \\
-15 & -5 & \frac{7}{2} & 0 & \frac{25}{2} & 25
\end{array}\right] \\
& A^{(4)}=\left[\begin{array}{rrrrrrrrrrrrr}
0 & 0 & 0 & 0 & 0 & 1 & 0 & 0 & 0 & 0 & 0 & 0 & 0 \\
2 & 0 & 0 & 0 & 0 & -3 & 0 & 0 & 0 & 0 & 0 & 0 & 0 \\
2 & -2 & 3 & 8 & -4 & -2 & 8 & -8 & -4 & 0 & -2 & 4 & -2 \\
0 & 2 & -4 & -4 & -4 & 1 & -4 & 4 & 8 & 0 & 4 & -8 & 4 \\
-2 & 2 & 0 & 4 & 4 & -1 & 4 & -4 & -8 & 0 & -4 & 8 & -4 \\
-30 & 50 & -100 & -160 & -40 & 76 & -100 & 100 & 200 & -120 & 100 & 100 & 25 \\
16 & -25 & 0 & -20 & -20 & -34 & 100 & 50 & 100 & 120 & -100 & -100 & -25 \\
-75 & 100 & 175 & 130 & 70 & 47 & -200 & -175 & -350 & -240 & 200 & 200 & 50 \\
-1 & 0 & 0 & -2 & 2 & 1 & 0 & 0 & 0 & 0 & 0 & 0 & 0 \\
-5 / 2 & 0 & 0 & -5 & 5 & 1 & 0 & 0 & 0 & 15 & 0 & 0 & 0 \\
-1 / 2 & 0 & 0 & 5 & 5 & -3 & 0 & 0 & 0 & -5 & 0 & 0 & 0 \\
33 / 2 & -10 & 20 & 53 & -13 & -29 & 20 & -20 & -40 & -15 & -20 & 10 & 10 \\
-1 / 2 & -10 & 0 & -11 & 1 & -7 & 40 & 20 & -20 & 15 & 20 & -10 & -10
\end{array}\right]
\end{aligned}
$$

References

[1] For a review see, for example: Johnson, D. L., 1983, J. Chim. phys., 80, 45.

[2] Stegemeyer, H., Blümel, Th., Hiltrop, K., Onussei, H., and Porsch, F., 1986, Liq. Crystals, $1,3$. 
[3] For a review see, for example: GramsBergen, E. F., Longa, L., and De Jeu, W. H., 1986, Phys. Rep., 135, 195.

[4] OseEn, C. W., 1933, Trans. Faraday Soc., 29, 883.

[5] Zocher, H., 1933, Trans. Faraday Soc., $29,945$.

[6] Frank, F. C., 1958, Discuss. Faraday Soc., 25, 19.

[7] Warren, W. E., 1970, Q. Jl Mech. appl. Math., 23, 525.

[8] Meiboom, S., Sammon, M., and Brinkman, and W. and F., 1983, Phys. Rev. A, 27, 438.

[9] ERICKSEN, J. L., 1966, Physics Fluids, 9, 1205.

[10] De Jeu, W. H., Classen, W. A. P., and Spruijt, A. M. J., 1976, Molec. Crystals liq. Crystals, 37, 269.

[11] Raynes, E. P., Tough, R. J. A., and Davies, K. A., 1979, Molec. Crystals liq. Crystals, $56,63$.

[12] De Jeu, W. H., 1980, Physical Properties of Liquid Crystalline Materials (Gordon \& Breach).

[13] Nehring, J., and Saupe A., 1971, J. chem. Phys., 54, 337.

[14] De Gennes, P. G., 1971, Molec. Crystals liq. Crystals, 12, 193. See also: De Gennes, P. G., 1974, The Physics of Liquid Crystals (Oxford University Press).

[15] Lubensky, T. C., 1970, Phys. Rev. A, 2, 2497.

[16] Schiele, K., and Trimper, S., 1983, Phys. Stat. Sol. B, 118, 267.

[17] Berreman, D. W., and Meiboom, S., 1984, Phys. Rev. A, 30, 1955.

[18] Poniewierski, A., and Sluckin, T. J., 1985, Molec. Phys., 55, 1113.

[19] Rościszewski, K., 1983, Acta phys. pol. A, 64, 277.

[20] Meyer, R. B., 1969, Phys. Rev. Lett., 22, 918. See also: Helfrich, W., 1971, Z. Naturf. (a), 26, 833.

[21] For a review, see: Hardonin, F., Levelut, A. M., Sigraud, G., Archard, M. F., Nguyen Mun Tinh, and Gasparoux, H., 1981, Symmetries and Broken Symmetries (Proceedings of the International Colloquium Pierre Curie), edited by N. Bocarra (IDSET).

[22] Barbero, G., Dozov, I., Palierne, J. F., and Durand, G., 1986, Phys. Rev. Lett., 56, 2056.

[23] Michelson, A., Benguigui, L., and Cabib, D., 1977, Phys. Rev. A, 16, 364.

[24] BLINC, R., 1977, Ferroelectrics, 16, 33.

[25] Khachaturyan, A. G., 1975, J. Phys. Chem. Solids, 36, 1055.

[26] Brand, H. R., 1986, Molec. Crystals liq. Crystals Lett., 35, 147.

[27] Sethna, J. P., Wright, D. C., and Mermin, N. D., 1983, Phys. Rev. Lett., 51, 467.

[28] Амгт, D. J., 1978, Field Theory: The Renormalization Group and Critical Phenomena (McGraw-Hill).

[29] EDmonds, A. R., 1957, Angular Momentum in Quantum Mechanics (Princeton University Press).

[30] LoNGA, L. (in preparation).

[31] Lyuksyutov, I. F., 1978, Soviet Phys. JETP, 48, 178. 